Article

\title{
Novel Fluorinated 7-Hydroxycoumarin Derivatives Containing an Oxime Ether Moiety: Design, Synthesis, Crystal Structure and Biological Evaluation
}

\author{
Qing-Qing Wang, Shu-Guang Zhang, Jian Jiao, Peng Dai and Wei-Hua Zhang *
}

check for updates

Citation: Wang, Q.-Q.; Zhang, S.-G.; Jiao, J.; Dai, P.; Zhang, W.-H. Novel Fluorinated 7-Hydroxycoumarin Derivatives Containing an Oxime Ether Moiety: Design, Synthesis, Crystal Structure and Biological Evaluation. Molecules 2021, 26, 372. https://doi.org/10.3390/molecules 26020372

Received: 8 December 2020 Accepted: 28 December 2020 Published: 12 January 2021

Publisher's Note: MDPI stays neutral with regard to jurisdictional clai$\mathrm{ms}$ in published maps and institutional affiliations.

Copyright: (C) 2021 by the authors. Licensee MDPI, Basel, Switzerland. This article is an open access article distributed under the terms and conditions of the Creative Commons Attribution (CC BY) license (https:// creativecommons.org/licenses/by/ $4.0 /)$.
Jiangsu Key Laboratory of Pesticide Science, College of Sciences, Nanjing Agricultural University, Nanjing 210095, China; 2017211001@njau.edu.cn (Q.-Q.W.); shuguangz@njau.edu.cn (S.-G.Z.); 2018211005@njau.edu.cn (J.J.); 2018211004@njau.edu.cn (P.D.)

* Correspondence: zhangweihua@njau.edu.cn; Tel.: +86-025-8439-5255

Abstract: A series of fluorinated 7-hydroxycoumarin derivatives containing an oxime ether moiety have been designed, synthesized and evaluated for their antifungal activity. All the target compounds were determined by ${ }^{1} \mathrm{H}-\mathrm{NMR},{ }^{13} \mathrm{C}-\mathrm{NMR}, \mathrm{FTIR}$ and HR-MS spectra. The single-crystal structures of compounds $4 \mathbf{e}, 4 \mathrm{~h}, \mathbf{5 h}$ and $\mathbf{6 c}$ were further confirmed using X-ray diffraction. The antifungal activities against Botrytis cinerea (B. cinerea), Alternaria solani (A. solani), Gibberella zeae (G. zeae), Rhizoctorzia solani (R. solani), Colletotrichum orbiculare (C. orbiculare) and Alternaria alternata (A. alternata) were evaluated in vitro. The preliminary bioassays showed that some of the designed compounds displayed the promising antifungal activities against the above tested fungi. Strikingly, the target compounds $\mathbf{5 f}$ and $\mathbf{6 h}$ exhibited outstanding antifungal activity against B. cinerea at $100 \mu \mathrm{g} / \mathrm{mL}$, with the corresponding inhibition rates reached 90.1 and $85.0 \%$, which were better than the positive control Osthole (83.6\%) and Azoxystrobin (46.5\%). The compound $5 \mathbf{f}$ was identified as the promising fungicide candidate against $B$. cinerea with the $\mathrm{EC}_{50}$ values of $5.75 \mu \mathrm{g} / \mathrm{mL}$, which was obviously better than Osthole $(33.20 \mu \mathrm{g} / \mathrm{mL})$ and Azoxystrobin $(64.95 \mu \mathrm{g} / \mathrm{mL})$. Meanwhile, the compound $5 \mathbf{f}$ showed remarkable antifungal activities against $R$. solani with the $E_{50}$ values of $28.96 \mu \mathrm{g} / \mathrm{mL}$, which was better than Osthole $(67.18 \mu \mathrm{g} / \mathrm{mL})$ and equivalent to Azoxystrobin $(21.34 \mu \mathrm{g} / \mathrm{mL})$. The results provide a significant foundation for the search of novel fluorinated 7-hydroxycoumarin derivatives with good antifungal activity.

Keywords: fluorinated 7-hydroxycoumarin; oxime ether derivatives; synthesis; crystal structure; antifungal activity

\section{Introduction}

The rapid growth of the world population will be a great challenge to obtain the enough food produced by limited arable lands [1]. In addition, the phytopathogenic fungi have emerged as the destructive parasites that results inevitably in large reductions in the yields and quality of agricultural products [2], thus seriously hindering the sustainable development of agriculture [3,4]. Under these circumstances, the rational application of agricultural fungicides has been identified as a most effective way to protect crops and to elevate agriculture yields [5]. However, fungicide-resistance problems have appeared in recent years due to the unreasonable utilization of existing antifungal agents for a long time, which leads to a series of side-effects including environmental pollution and food safety threats [6,7]. Therefore, it is of great significance to develop novel high-efficiency and broad-spectrum fungicides.

Coumarin derivatives, the typical natural products containing benzopyrone structures, have widely served as secondary metabolites in plants [8,9]. Interestingly, the natural coumarins exhibit the noticeable bioactivities including antitumor, anticoagulant, anti-inflammatory, antioxidant, antifungal and antiviral effects [10-12]. In view of this, 
coumarin derivatives have been widely applied to agricultural and medicinal chemistries. The corresponding synthesis methods and pharmacological activities were also well reported, such as Osthole, Coumoxystrobin and Warfarin (Figure 1). Employing Osthole as a lead structure, our group synthesized a series of coumarin derivatives against the phytopathogenic fungi effectively (Figure 2), including coumarin [8,7-e][1,3]oxazine [13], furo[3,2-c]coumarin [14], pyrano[3,2-c]chromene-2,5-dione [14], coumarin-3-carboxamide derivatives [15], coumarin ring-opening derivatives [16] and pyrrole/pyrazole-substituted coumarin derivatives [17]. Due to the pharmacological diversity of coumarin derivatives, it has been described as one of "privileged scaffolds" [18,19]. Obviously, the diversified derivatization of the coumarin parent ring is an important strategy for the development of highly effective compounds, making coumarin a candidate for the development of more efficient compounds.

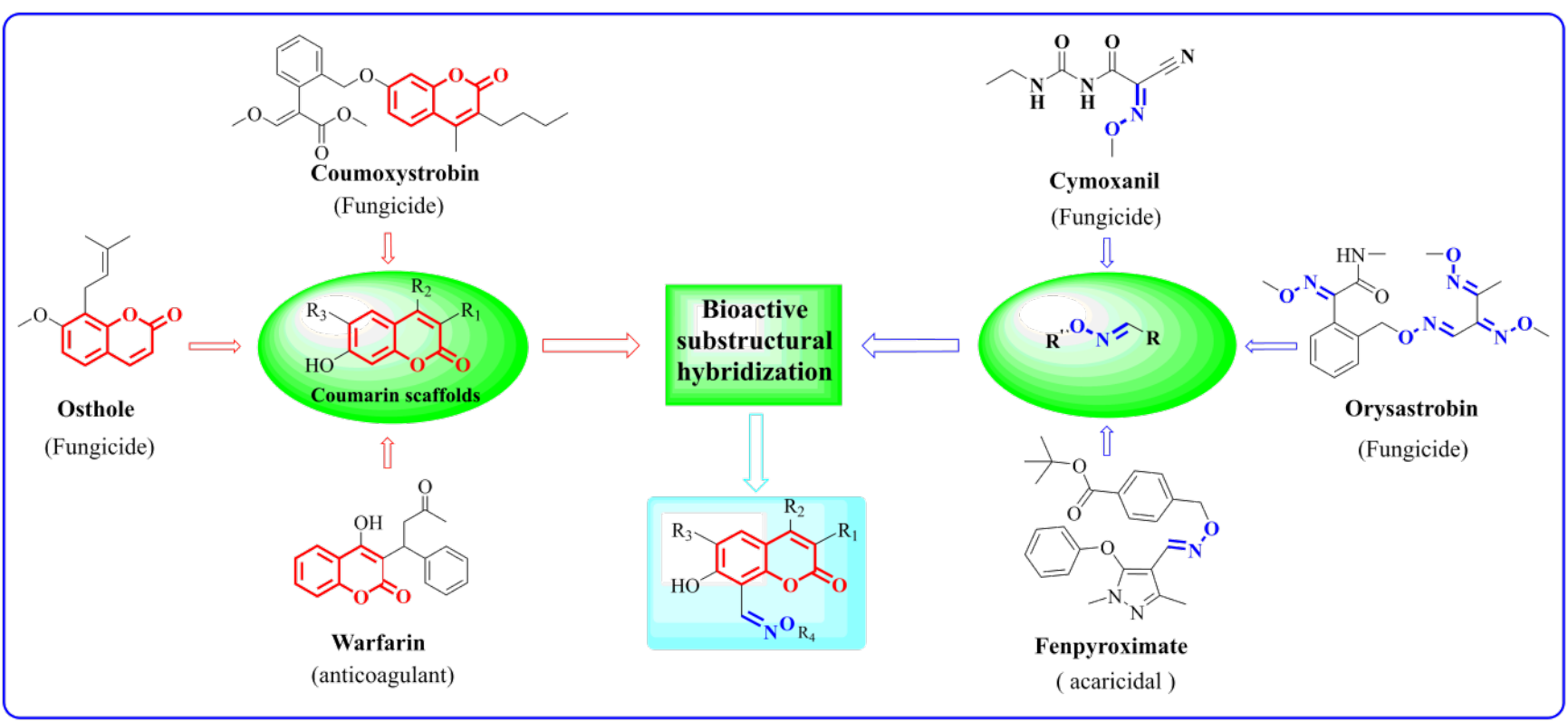

Figure 1. Design strategy of target 7-hydroxycoumarin oxime ether structures.<smiles>O=c1cc(C(F)(F)F)c2cc(Cl)c3c(c2o1)CN(c1ccccc1)CO3</smiles>

coumarino $[8,7-e][1,3]$ oxazine<smiles>O=C(NNc1ccc(Cl)cc1)c1cc2ccccc2oc1=O</smiles>

coumarin-3-carboxamide derivatives<smiles>C=CCOc1ccc2c(c1)oc(=O)c1c(C)c(C(=O)OC)oc12</smiles>

furo[3,2-c]coumarin<smiles>COC(=O)c1c(-c2ccccc2OC)oc(C)c1C</smiles>

coumarin ring-opening derivatives<smiles>CCc1c(C)c2c(=O)oc3cc(OC)ccc3c2oc1=O</smiles>

pyrano[3,2-c] chromene-2,5-dione<smiles>O=c1cc(C(F)(F)F)c2ccc(-n3cccn3)cc2o1</smiles>

7-pyrazolecoumarins

Figure 2. Our group's previous work on coumarin derivatives. 
in Scheme 1. The reaction progress was monitored using TLC $\left(\mathrm{V}_{\text {ethyl acetate }} / \mathrm{V}_{\text {petroleum ether }}\right.$ $=1: 2$ ). The desired target compounds were obtained by column chromatography from a mixed solution of ethyl acetate/petroleum ether $\left(\mathrm{V}_{\text {petroleum ether }}: \mathrm{V}_{\text {ethyl acetate }}=1: 20\right)$, with yields of $17 \%$ to $83 \%$. To the best of our knowledge, there have been no report on the production of oxime ether derivatives of 7-hydroxycoumarin at 8-position $[33,34]$. The structures of all the synthetic coumarin derivatives were confirmed by ${ }^{1} \mathrm{H}-\mathrm{NMR},{ }^{13} \mathrm{C}-\mathrm{NMR}$, FTIR and HRMS spectral data. The data of target compound $5 \mathrm{~h}$ was analyzed as a representative example. The ${ }^{1} \mathrm{H}-\mathrm{NMR}$ spectrum of compound $5 \mathbf{h}$ showed two singlets at 11.53 and $8.88 \mathrm{ppm}$, which meant the molecular structure of compound $5 \mathrm{~h}$ had a $-\mathrm{OH}$ group and $-\mathrm{CH}=\mathrm{N}$ - group. The doublet at $4.82 \mathrm{ppm}$ and the triplet at $2.59 \mathrm{ppm}$ were attributed to the presence of propynyl. In the ${ }^{13} \mathrm{C}-\mathrm{NMR}$ spectrum, a signal peak at $157.46 \mathrm{ppm}$ indicated that target compound $5 \mathrm{~h}$ had a carbonyl group. The quadruple peaks at 141.04 and $113.18 \mathrm{ppm}$ were assigned to $-\mathrm{CF}_{3}$ group. Meanwhile, three singles at 77.65, 76.40 and $62.81 \mathrm{ppm}$ confirmed the existence of propynyl group in the structure of compound $5 \mathrm{~h}$. In the FTIR spectrum of compound $5 \mathrm{~h}$, the absorption peak of alkynyl group appears at $2131.6 \mathrm{~cm}^{-1}$ and the strong peak at $1736.3 \mathrm{~cm}^{-1}$ indicated the presence of carbonyl group. In the HRMS spectrum of compounds $5 \mathrm{~h}$, the value of $[\mathrm{M}+\mathrm{H}]^{+}$ion absorption signal was 346.0088, which was consistent with the calculated value (346.0086) for $\mathrm{C}_{14} \mathrm{H}_{7} \mathrm{ClF}_{3} \mathrm{NO}_{4}[\mathrm{M}+\mathrm{H}]^{+}$.

\subsection{Single Crystal Structures of Compounds $4 \mathbf{e}, 4 \mathrm{~h}, 5 \mathrm{~h}$ and $6 \mathrm{c}$}

To further understand the actual structure of synthesized compounds, the structures of compound $4 \mathrm{e}, 4 \mathrm{~h}, 5 \mathrm{~h}$ and $6 \mathrm{c}$ were determined as representative examples by X-ray diffraction analysis. The tested single crystals were crystallized from the methanol containing the title compounds under room temperature until the size of the crystals was enough to test. The corresponding crystal structure diagram and crystal packing diagram were presented in Figure 3.

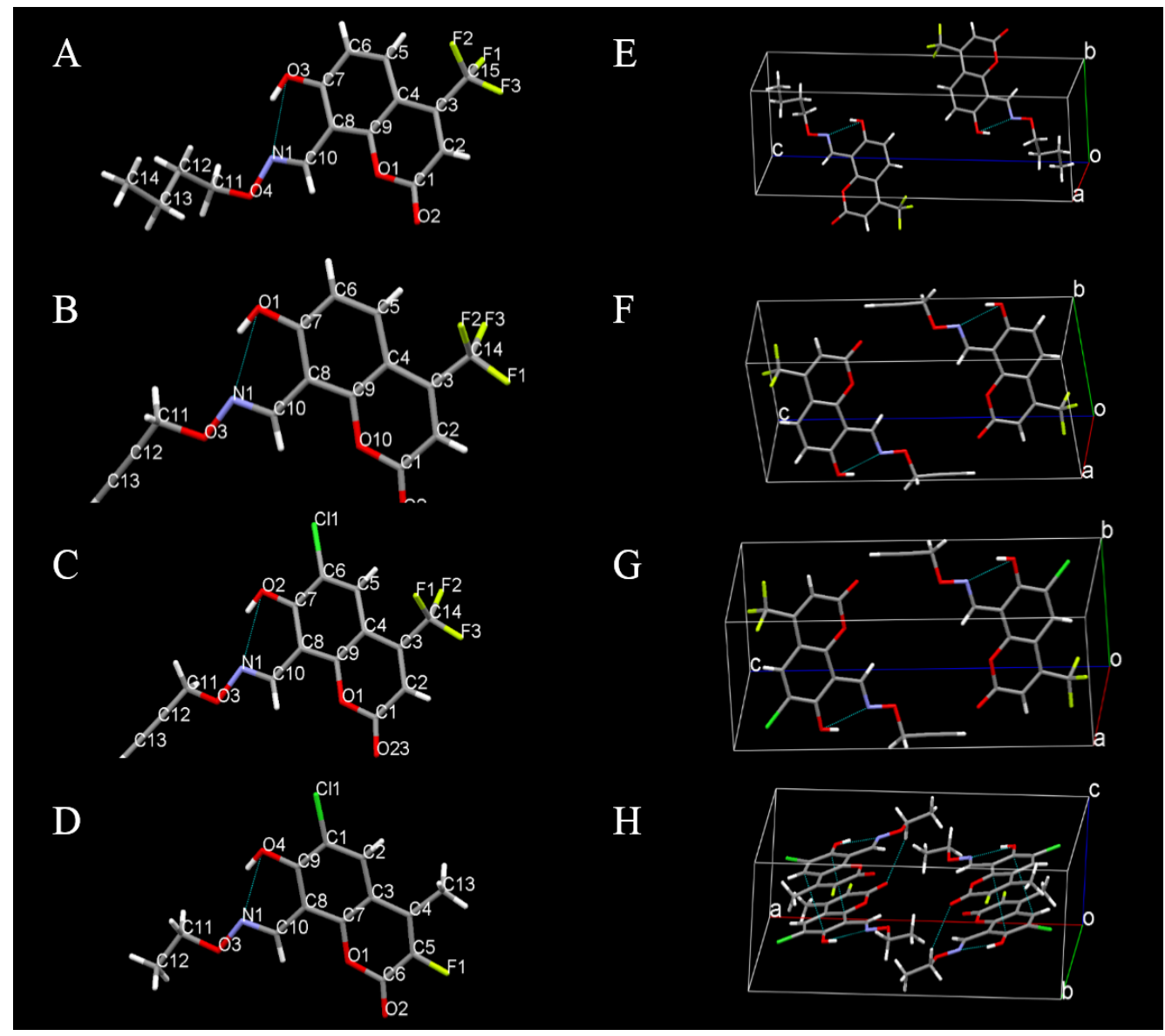

Figure 3. (A-D) The crystal structural diagram of $4 \mathrm{e}, 4 \mathrm{~h}, 5 \mathrm{~h}$ and $6 \mathrm{c}$. (E-H) The corresponding crystal packing diagram of $4 \mathrm{e}, 4 \mathrm{~h}, 5 \mathrm{~h}$ and $6 \mathrm{c}$. 


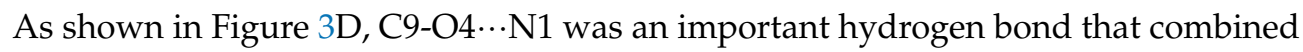
with 7-hydroxy-2H-chromen-2-one and oxime ether fragment which construct the main scaffold of $\mathbf{6 c}$. From this figure we could also see that these four compounds had trans structure, which might be owing to the low energy and stability of the trans structure.

As shown in Figure $3 \mathrm{H}$, the intermolecular $\pi-\pi$ conjugation force and hydrogen bonds between neighboring molecules were primary force for establishing the three-dimensional structure of compound $\mathbf{6 c}$. The crystallographic data of compound $\mathbf{6 c}$ was deposited at the Cambridge Crystallographic Data Centre with an assigned number of CCDC 2043279, the crystallographic data were listed in Table 1 . The CCDC numbers of $4 \mathbf{e}, 4 \mathbf{h}$ and $5 \mathbf{h}$ were 2043278, 2043275 and 2043276, respectively. The corresponding crystallographic data were listed in Tables S1-S3 (Supplementary Materials).

Table 1. Crystal data of title compound $\mathbf{6 c}$.

\begin{tabular}{|c|c|}
\hline \multicolumn{2}{|c|}{ Crystal Data } \\
\hline CCDC Number & 2043279 \\
\hline $\mathrm{C}_{13} \mathrm{H}_{11} \mathrm{ClFNO}_{4}$ & $\alpha=90^{\circ}$ \\
\hline $\mathrm{FW}=299.68$ & $\beta=94.286(10)^{\circ}$ \\
\hline Triclinic, $P 21$ & $\gamma=90^{\circ}$ \\
\hline$a=18.195(2) \AA$ & $V=1320.7(2) \AA^{3}$ \\
\hline$b=10.5938(7) \AA$ & $Z=4$ \\
\hline$c=6.8711(9) \AA$ & $\mu=0.314 \mathrm{~mm}^{-1}$ \\
\hline$F(000)=616$ & Crystal size $\left(\mathrm{mm}^{3}\right): 0.21 \times 0.12 \times 0.08$ \\
\hline \multicolumn{2}{|c|}{ Data Collection } \\
\hline 3400 observed reflections with $I>2 \sigma(I)$ & Radiation $(\AA): \operatorname{MoK} \alpha(\lambda=0.71073)$ \\
\hline 7579 measured reflections & $R_{\text {int }}=0.062$ \\
\hline 2329 independent reflections & $\theta_{\min }=2.3^{\circ}, \theta_{\max }=25.03^{\circ}$ \\
\hline \multicolumn{2}{|c|}{ Refinement } \\
\hline 184 parameters & $S=0.919$ \\
\hline 2327 reflections & $R\left[F^{2}>2 \sigma\left(F^{2}\right)\right]=0.0486$ \\
\hline
\end{tabular}

\subsection{In Vitro Antifungal Activity of Target Compounds}

The in vitro antifungal activity was evaluated by a mycelium growth rate method and the corresponding results were listed in Table 2. Osthole and Azoxystrobin were used as the positive control fungicides throughout the experiment. Noteworthy, the antifungal activities of target compounds against $B$. cinerea and $R$. solan $i$ were generally better than that of A. solani, G. zeae, C. orbiculare and A. alternata. Especially, the target compounds 5f and $6 \mathrm{~h}$ exhibited outstanding antifungal activity against $B$. cinerea at $100 \mu \mathrm{g} / \mathrm{mL}$ with the corresponding inhibition rates of 90.1 and $85.0 \%$, which were superior to the positive control fungicides Osthole (83.6\%) and Azoxystrobin (46.5\%). Meanwhile, the intermediate compounds $\mathbf{2 a}, \mathbf{2 b}$ and target compounds $\mathbf{4 f}, \mathbf{5 a}, \mathbf{5 f}$ and $\mathbf{6 f}$ showed remarkable antifungal activities against $R$. solani at $100 \mu \mathrm{g} / \mathrm{mL}$ with the corresponding inhibition rates of 86.2, $64.6,71.7,64.6,72.6$ and $70.1 \%$, which were equivalent to the positive control fungicides Osthole (82.7\%) and Azoxystrobin (72.3\%). Unfortunately, the partial target compounds were not sensitive to $A$. solani and G. zeae. 
Table 2. Antifungal effects of target compounds at $100 \mu \mathrm{g} / \mathrm{mL}$.

\begin{tabular}{|c|c|c|c|c|c|c|}
\hline \multirow{2}{*}{ Compound } & \multicolumn{6}{|c|}{ Inhibition (\%) } \\
\hline & B. cinerea & A. solani & G. zeae & R. solani & C. orbiculare & A. alternata \\
\hline $2 a$ & $50.0^{a}$ & 60.8 & 50.0 & 86.2 & 45.3 & 39.7 \\
\hline $2 b$ & 77.5 & 51.7 & 57.8 & 64.6 & 56.3 & 64.1 \\
\hline $2 c$ & $10.0^{\mathrm{b}}$ & 10.0 & 10.0 & 35.1 & 10.0 & 18.3 \\
\hline $4 a$ & 37.5 & 15.0 & 33.3 & 38.5 & 53.1 & 27.0 \\
\hline $4 b$ & 12.5 & 10.0 & 10.0 & 10.0 & 10.0 & 10.0 \\
\hline $4 c$ & 18.8 & 10.0 & 10.0 & 12.3 & 14.1 & 10.0 \\
\hline $4 d$ & 13.5 & 10.0 & 10.0 & 10.0 & 12.5 & 10.0 \\
\hline $4 e$ & 18.8 & 10.0 & 10.0 & 10.0 & 10.0 & 10.0 \\
\hline $4 f$ & 10.0 & 10.0 & 10.0 & 71.7 & 29.7 & 10.0 \\
\hline $4 g$ & 10.0 & 10.0 & 11.4 & 10.0 & 23.0 & 10.0 \\
\hline $4 \mathrm{~h}$ & 15.4 & 10.0 & 10.0 & 10.0 & 10.0 & 10.0 \\
\hline $4 i$ & 10.0 & 10.0 & 10.0 & 47.2 & 14.1 & 10.0 \\
\hline $4 j$ & 10.0 & 10.0 & 10.0 & 10.0 & 10.0 & 10.0 \\
\hline $5 a$ & 62.5 & 23.3 & 37.5 & 64.6 & 55.6 & 46.5 \\
\hline $5 b$ & 21.3 & 10.0 & 10.0 & 16.9 & 17.2 & 10.0 \\
\hline $5 c$ & 12.5 & 10.0 & 10.0 & 16.9 & 14.1 & 10.0 \\
\hline $5 d$ & 18.8 & 10.0 & 10.0 & 24.6 & 10.0 & 10.0 \\
\hline $5 e$ & 10.0 & 10.0 & 10.0 & 10.0 & 14.1 & 10.0 \\
\hline $5 f$ & 90.1 & 33.0 & 58.7 & 72.6 & 46.9 & 10.8 \\
\hline $5 g$ & 10.8 & 10.0 & 10.0 & 23.8 & 12.3 & 10.0 \\
\hline $5 \mathrm{~h}$ & 10.0 & 10.0 & 10.0 & 10.0 & 10.0 & 10.0 \\
\hline $5 i$ & 10.0 & 10.0 & 10.0 & 28.3 & 10.0 & 10.0 \\
\hline $5 j$ & 27.7 & 10.0 & 10.0 & 11.2 & 10.0 & 10.0 \\
\hline $6 a$ & 58.9 & 29.8 & 26.0 & 49.1 & 51.1 & 51.5 \\
\hline $6 b$ & 29.8 & 10.0 & 17.6 & 36.4 & 19.6 & 17.6 \\
\hline $6 c$ & 29.8 & 10.0 & 10.2 & 10.0 & 16.0 & 10.0 \\
\hline $6 d$ & 38.6 & 10.0 & 10.0 & 10.0 & 10.7 & 10.0 \\
\hline $6 e$ & 29.8 & 10.0 & 10.0 & 27.3 & 19.6 & 14.7 \\
\hline $6 f$ & 10.0 & 20.5 & 13.3 & 70.1 & 32.8 & 10.0 \\
\hline $6 g$ & 13.8 & 10.0 & 17.1 & 10.0 & 13.7 & 10.0 \\
\hline $6 \mathrm{~h}$ & 85.0 & 20.6 & 32.9 & 53.8 & 58.5 & 10.9 \\
\hline $6 i$ & 20.6 & 10.0 & 10.0 & 41.7 & 20.3 & 10.0 \\
\hline $6 j$ & 39.2 & 10.0 & 36.2 & 28.3 & 10.0 & 10.0 \\
\hline Osthole & 83.6 & 40.0 & 56.7 & 82.7 & 16.7 & 22.4 \\
\hline Azoxystrobin & 76.5 & 30.5 & 71.7 & 72.3 & 94.5 & 61.1 \\
\hline
\end{tabular}

average value of three replications; ${ }^{\mathrm{b}} 10.0$ indicated the data below $10 \%$ inhibitory ratio.

To better investigate the inhibitory performance of target compounds against phytopathogenic fungi, the compounds that exhibited the inhibition rates exceeding $60 \%$ at $100 \mu \mathrm{g} / \mathrm{mL}$ were further tested for their $\mathrm{EC}_{50}$ values. Osthole and Azoxystrobin were served as the positive control. As exhibited in Table 3, the $\mathrm{EC}_{50}$ values of target compounds 5f and $\mathbf{6 h}$ were notably as low as $5.75 \mu \mathrm{g} / \mathrm{mL}$ and $13.75 \mu \mathrm{g} / \mathrm{mL}$, proving that they were much more effective than Osthole $(33.20 \mu \mathrm{g} / \mathrm{mL})$ and Azoxystrobin $(64.95 \mu \mathrm{g} / \mathrm{mL})$ against B. cinerea. Meanwhile, as listed in Table 4, the $\mathrm{EC}_{50}$ values of all the detected compounds were lower than Osthole $(67.18 \mu \mathrm{g} / \mathrm{mL})$ against $R$. solani. Specifically, the $\mathrm{EC}_{50}$ values of the target compounds $\mathbf{2 b}, \mathbf{4 f}, \mathbf{5 a}, \mathbf{5 f}$ and $\mathbf{6 f}$ were $17.72,7.48,33.10,28.96$ and $28.70 \mu \mathrm{g} / \mathrm{mL}$, respectively. The corresponding in vitro antifungal performances of compound $\mathbf{5 f}$, Osthole and Azoxystrobin against B. cinerea were presented in Figure 4, which visually exhibited the corresponding in vitro inhibition of $B$. cinerea. 
Table 3. $\mathrm{EC}_{50}$ values determination of some target compounds against B. cinerea.

\begin{tabular}{ccccc}
\hline Compound & Toxic Regression & $\mathbf{R}$ & EC $_{\mathbf{5 0}}(\boldsymbol{\mu g} / \mathbf{m L})$ & EC $_{\mathbf{5 0}}(\boldsymbol{\mu M})$ \\
\hline $\mathbf{2 b}$ & $\mathrm{y}=2.02 \mathrm{x}+1.59$ & 0.99 & 47.73 & 163.12 \\
$\mathbf{5 a}$ & $\mathrm{y}=1.30 \mathrm{x}+3.27$ & 0.99 & 13.25 & 43.07 \\
$\mathbf{5 f}$ & $\mathrm{y}=0.35 \mathrm{x}+4.74$ & 0.98 & 5.75 & 16.54 \\
$\mathbf{6 h}$ & $\mathrm{y}=0.93 \mathrm{x}+3.93$ & 0.98 & 13.75 & 44.40 \\
Osthole & $\mathrm{y}=1.31 \mathrm{x}+3.00$ & 0.99 & 33.20 & 148.02 \\
Azoxystrobin & $\mathrm{y}=1.06 \mathrm{x}+3.10$ & 0.99 & 64.95 & 161.01 \\
\hline
\end{tabular}

The $\mathrm{EC}_{50}$ value was the average value of three replications.

Table 4. $\mathrm{EC}_{50}$ values determination of some target compounds against $R$. solani.

\begin{tabular}{ccccc}
\hline Compound & Toxic Regression & $\mathbf{R}$ & EC $_{\mathbf{5 0}}(\boldsymbol{\mu g} / \mathbf{m L})$ & EC $_{\mathbf{5 0}}(\boldsymbol{\mu M})$ \\
\hline $\mathbf{2 b}$ & $\mathrm{y}=1.50 \mathrm{x}+3.12$ & 0.99 & 17.72 & 60.56 \\
$\mathbf{4 f}$ & $\mathrm{y}=0.53 \mathrm{x}+4.53$ & 0.91 & 7.48 & 23.88 \\
$\mathbf{5 a}$ & $\mathrm{y}=1.26 \mathrm{x}+3.07$ & 0.99 & 33.10 & 107.60 \\
$\mathbf{5 f}$ & $\mathrm{y}=0.45 \mathrm{x}+4.34$ & 0.96 & 28.96 & 83.30 \\
$\mathbf{6 f}$ & $\mathrm{y}=1.19 \mathrm{x}+3.26$ & 0.99 & 28.70 & 92.08 \\
Osthole & $\mathrm{y}=0.95 \mathrm{x}+3.25$ & 0.99 & 67.18 & 299.52 \\
Azoxystrobin & $\mathrm{y}=0.86 \mathrm{x}+3.80$ & 0.99 & 21.34 & 52.90 \\
\hline
\end{tabular}

The $\mathrm{EC}_{50}$ value was the average value of three replications.

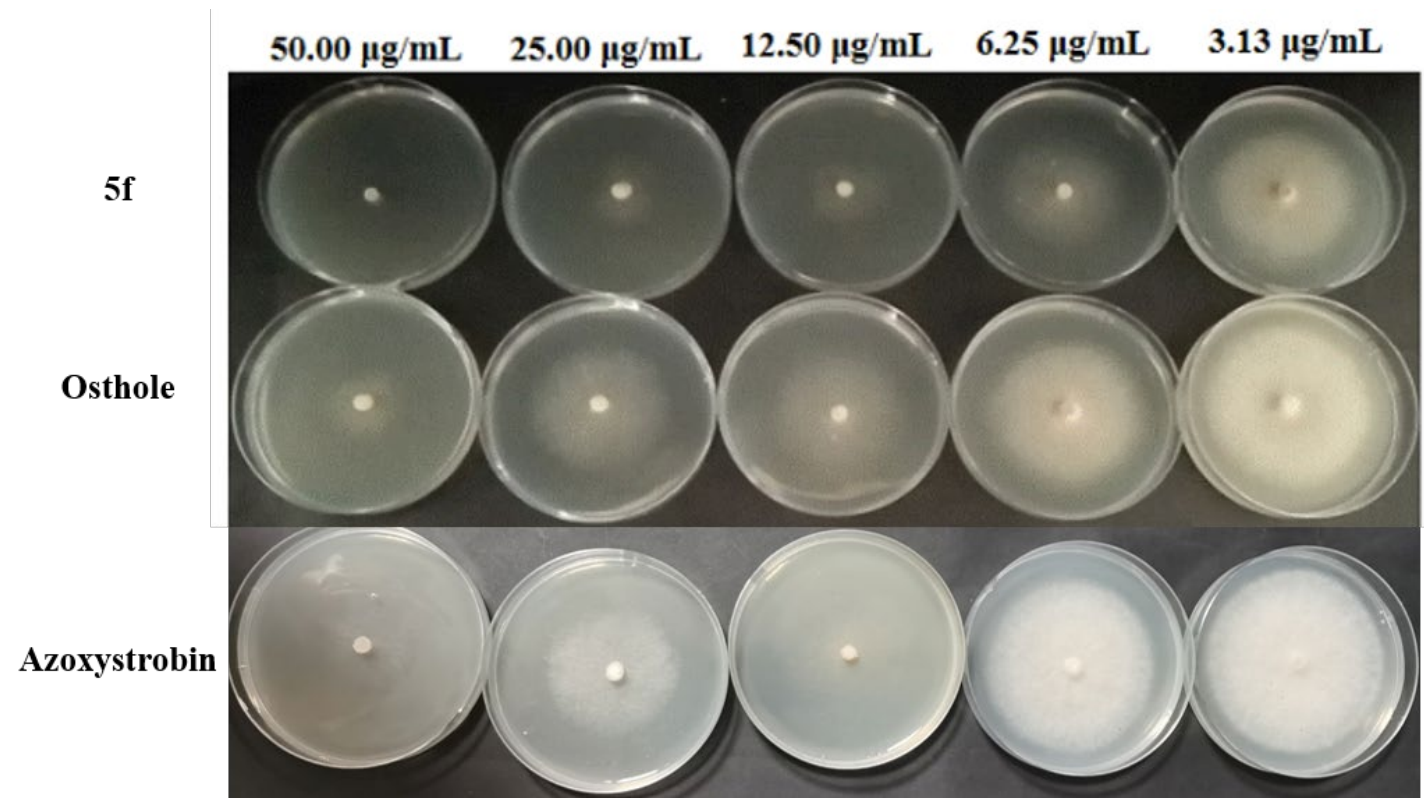

Figure 4. In vitro inhibition of mycelial growth of B. cinerea by compound 5f, Osthole and Azoxystrobin.

\subsection{Structure-Activity Relationships}

The bioassay results in Tables 2-4 indicated that the antifungal activities of most synthesized 7-hydroxycoumarin oxime derivatives were certain poor, which made it difficult to extract a clear structure-activity relationship analysis. Nevertheless, some broad conclusions could still be drawn. First, the most target compounds were noticeably more efficient against B. cinerea and $R$. solani than A. solani, G. zeae, C. orbiculare and A. alternata. Second, compounds $4 \mathbf{a}, \mathbf{5 a}, \mathbf{5 f}, \mathbf{6} \mathbf{a}$ and $\mathbf{6 h}$ displayed the preferable activity than the positive control Osthole against $C$. orbiculare. Meanwhile, compound $\mathbf{6 a}$ also possessed more effective activity against $A$. alternata than Osthole. Third, the antifungal activities generally could be improved if $\mathrm{R}_{3}$ was $\mathrm{Cl}$ and $\mathrm{R}_{4}$ was $\mathrm{H}$ atom, allyl or propargyl, highlighted by $\mathbf{4 f}, \mathbf{5 a}, \mathbf{5 f}, \mathbf{6 a}$, 
6f and $\mathbf{6 h}$, all of which displayed a broad spectrum of antifungal activity against $B$. cinerea and $R$. solani. Forth, we still could find that the intermediate compounds $\mathbf{2} \mathbf{a}$ and $\mathbf{2} \mathbf{b}$ showed a broad antifungal spectrum, which could be served as the active candidates for structure optimization.

\section{Experimental Section}

\subsection{Chemicals and Instruments}

Resorcin, 4-chlororesorcinol, ethyl 4,4,4-trifluoroacetoacetate and ethyl 2-fluoroacetoacetate were obtained from Sinopharm Chemical Reagent Co. Ltd., Shanghai, China. Hydroxylamine hydrochloride, hexamethylenetetramine and trifluoroacetic acid were purchased from Aladdin Reagent Co. Ltd., Shanghai, China. All other chemicals were commercially available and used without further purification. The progress of reactions and the purity of products were monitored by TLC using silica gel GF/UV 254. The melting points of coumarin compounds were measured on an X-4 apparatus (uncorrected). Fourier transform infrared (FTIR) spectra were recorded on a Bruker Tensor 27 spectrometer (Billerica, MA, USA), and all samples were prepared as $\mathrm{KBr}$ plates. ${ }^{1} \mathrm{H}-\mathrm{NMR}$ and ${ }^{13} \mathrm{C}-\mathrm{NMR}$ spectra were detected on a Bruker Avance $400 \mathrm{MHz}$ spectrometer with TMS as an internal standard. HR-MS (ESI) spectra were operated using a Thermo Exactive spectrometer (Waltham, MA, USA). X-rays were measured at $296 \mathrm{~K}$ on a Bruker SMART APEX2 CCD area detector diffractometer.

\subsection{Chemistry}

3.2.1. General Procedure for the Synthesis of the Intermediates 2

Fluorinated 7-hydroxycoumarins were synthesized through reported procedures [25]. Trifluoroacetic acid $(38 \mathrm{~mL})$ containing 7-hydroxycoumarin $(8.7 \mathrm{mmol})$ and hexamethylenetetramine $(43.5 \mathrm{mmol})$ was stirred at reflux for $1.5 \mathrm{~h}$. Then, aqueous hydrochloric acid $(30 \%, 75 \mathrm{~mL})$ was added into the mentioned-above reaction solution. After stirring $45 \mathrm{~min}$ under $70{ }^{\circ} \mathrm{C}$, the resulting mixture was quenched with ice water and extracted with ethyl acetate. The obtained organic layer was neutralized by sodium bicarbonate solution, washed with water, dried by anhydrous sodium sulfate and filtered. After concentrating under vacuum, the residue containing an intermediate $\mathbf{2}$ was purified by the column chromatography using ethyl acetate/petroleum ether $\left(\mathrm{V}_{\text {ethyl acetate }} / \mathrm{V}_{\text {petroleum ether }}=1: 2\right)$ as eluent.

7-hydroxy-4-(trifluoromethyl)-2H-chromen-2-one (1a): pink solid; mp 186.8-187.8 ${ }^{\circ} \mathrm{C}$; yield 54\%; ${ }^{1} \mathrm{H}-\mathrm{NMR}\left(400 \mathrm{MHz}, \mathrm{DMSO}-d_{6}\right) \delta 10.97(\mathrm{~s}, 1 \mathrm{H}), 7.53(\mathrm{~s}, 1 \mathrm{H}), 6.95-6.86(\mathrm{~m}, 1 \mathrm{H}), 6.81(\mathrm{~d}$, $J=2.3 \mathrm{~Hz}, 1 \mathrm{H}), 6.78-6.68(\mathrm{~m}, 1 \mathrm{H})$; IR $(\mathrm{KBr}) \vee\left(\mathrm{cm}^{-1}\right) 3419,3096,1703,1600,1409,1280,1127$, 866,842 .

6-chloro-7-hydroxy-4-(trifluoromethyl)-2H-chromen-2-one (1b): pink solid; mp 184.9-185.0 ${ }^{\circ} \mathrm{C}$; yield 34\%; ${ }^{1} \mathrm{H}-\mathrm{NMR}\left(400 \mathrm{MHz}, \mathrm{DMSO}-d_{6}\right) \delta 11.85$ (s, 1H), 7.57 (s, 1H), 7.02 (s, 1H), 6.88 $(\mathrm{s}, 1 \mathrm{H})$; IR $(\mathrm{KBr}) \vee\left(\mathrm{cm}^{-1}\right) 3595,3414,3096,1694,1598,1400,1280,1247,1145,866,836$.

6-chloro-3-fluoro-7-hydroxy-4-methyl-2H-chromen-2-one (1c): pink solid; mp 235.6-236.1 ${ }^{\circ} \mathrm{C}$; yield 41\%; ${ }^{1} \mathrm{H}-\mathrm{NMR}\left(400 \mathrm{MHz}\right.$, Acetone- $\left.d_{6}\right) \delta 9.73$ (s, 1H), 7.69 (s, 1H), $6.92(\mathrm{~s}, 1 \mathrm{H}), 2.41$ (s, $3 \mathrm{H})$; IR (KBr) v $\left(\mathrm{cm}^{-1}\right) 3071,1695,1603,1384,1302,1137,858,750$.

7-hydroxy-2-oxo-4-(trifluoromethyl)-2H-chromene-8-carbaldehyde (2a): white solid; mp 129.0$129 .{ }^{\circ} \mathrm{C}$; yield 44\%; ${ }^{1} \mathrm{H}-\mathrm{NMR}\left(400 \mathrm{MHz}, \mathrm{DMSO}-d_{6}\right) \delta 12.05(\mathrm{~s}, 1 \mathrm{H}), 10.39(\mathrm{~s}, 1 \mathrm{H}), 7.76(\mathrm{~d}$, $J=8.9 \mathrm{~Hz}, 1 \mathrm{H}), 7.01(\mathrm{~d}, J=9.1 \mathrm{~Hz}, 1 \mathrm{H}), 6.89(\mathrm{~s}, 1 \mathrm{H}) ;{ }^{13} \mathrm{C}-\mathrm{NMR}\left(101 \mathrm{MHz}, \mathrm{DMSO}-d_{6}\right) \delta$ $190.31,164.78,158.01,156.43,139.83(\mathrm{q}, J=32.5 \mathrm{~Hz}), 132.15,121.93$ (d, $J=275.6 \mathrm{~Hz}), 115.29$, $113.66(\mathrm{q}, J=4.0 \mathrm{~Hz}), 110.31,105.64 ; \mathrm{IR}(\mathrm{KBr}) \vee\left(\mathrm{cm}^{-1}\right)$ 3097, 2920, 1745, 1405, 1280; HR-MS (ESI): $m / z$ calcd. for $\mathrm{C}_{11} \mathrm{H}_{5} \mathrm{~F}_{3} \mathrm{O}_{4}\left([\mathrm{M}+\mathrm{H}]^{+}\right)$259.0140, found 259.0774.

6-chloro-7-hydroxy-2-oxo-4-(trifluoromethyl)-2H-chromene-8-carbaldehyde (2b): yellow solid; mp 164.9-165.7 ${ }^{\circ} \mathrm{C}$; yield 75\%; ${ }^{1} \mathrm{H}-\mathrm{NMR}\left(400 \mathrm{MHz}, \mathrm{DMSO}-d_{6}\right) \delta 11.79$ (s, 1H), 10.41 (s, 1H), 7.83 (s, 1H), 7.07 (s, 1H); ${ }^{13} \mathrm{C}-\mathrm{NMR}\left(101 \mathrm{MHz}, \mathrm{DMSO}-d_{6}\right) \delta 192.26,159.99,157.41$, 
$155.59,138.86(q, J=32.5 \mathrm{~Hz}), 130.75,121.71(\mathrm{~d}, J=275.3 \mathrm{~Hz}), 118.83,115.20(\mathrm{q}, J=4.7 \mathrm{~Hz})$, 110.64, 106.21; IR (KBr) v (cm $\left.{ }^{-1}\right)$ 3094, 2920, 1740, 1427, 1394, 1296; HR-MS (ESI): $\mathrm{m} / z$ calcd. for $\mathrm{C}_{11} \mathrm{H}_{4} \mathrm{ClF}_{3} \mathrm{O}_{4}\left([\mathrm{M}+\mathrm{H}]^{+}\right)$292.9750, found 293.0403 .

6-chloro-3-fluoro-7-hydroxy-4-methyl-2-oxo-2H-chromene-8-carbaldehyde (2c): yellow solid; mp 145.5-146.4 ${ }^{\circ} \mathrm{C}$; yield 48\%; ${ }^{1} \mathrm{H}-\mathrm{NMR}\left(400 \mathrm{MHz}, \mathrm{DMSO}-d_{6}\right) \delta 12.32$ (s, 1H), 10.42 (s, 1H), 8.20 (s, 1H), 2.39 (s, 3H); ${ }^{13}$ C-NMR (101 MHz, DMSO) $\delta 192.57$ (s), 158.46 (s), 153.56 (d, $J=30.8 \mathrm{~Hz}), 150.88(\mathrm{~d}, J=2.3 \mathrm{~Hz}), 142.59(\mathrm{~d}, J=245.2 \mathrm{~Hz}), 132.79(\mathrm{~d}, J=5.7 \mathrm{~Hz}), 132.00$ $(\mathrm{d}, J=13.9 \mathrm{~Hz}), 118.69$ (s), 112.39 (s), 109.95 (s), $10.62(\mathrm{~d}, J=3.2 \mathrm{~Hz}) . \mathrm{IR}(\mathrm{KBr}) v\left(\mathrm{~cm}^{-1}\right)$ 3095, 3031, 2952, 1723, 1613, 1302; HR-MS (ESI): $m / z$ calcd. for $\mathrm{C}_{11} \mathrm{H}_{6} \mathrm{ClFO}_{4}\left([\mathrm{M}+\mathrm{Na}]^{+}\right)$ 278.9939 , found 279.0235 .

\subsubsection{General Procedure for Synthesis of $O$-substituted hydroxylamines $\mathbf{3 d} \mathbf{d} \mathbf{3} \mathbf{j}$}

The water $(5 \mathrm{~mL})$ mixing hydroxylamine hydrochloride $(0.10 \mathrm{~mol})$, ethyl acetate $(0.18 \mathrm{~mol})$ and $28 \%$ sodium hydroxide aqueous solution $(25 \mathrm{~mL})$ were stirred at $0{ }^{\circ} \mathrm{C}$. The resultant mixture was then stirred at room temperature for $1 \mathrm{~h}$. After that, halogenated hydrocarbon $(0.11 \mathrm{~mol})$ was dropwise added and the reaction was stirred at $60^{\circ} \mathrm{C}$ for further $6 \mathrm{~h}$. Subsequently, the reaction mixture was extracted with petroleum ether and ethyl acetate. The organic layer was combined and dried by anhydrous sodium sulfate, then the solvent was removed under reduced pressure and oily product was obtained. The obtained oily product was dissolved in ethyl alcohol, and 36\% aqueous hydrochloric acid was added to the solution. The mixture was stirred at $50{ }^{\circ} \mathrm{C}$ for $4 \mathrm{~h}$. Finally, the reaction was neutralized to $\mathrm{pH} 7.0$ by saturated sodium bicarbonate aqueous solution, and then extracted with dichloromethane. The extracts were dried over anhydrous sodium sulfate, and concentrated under vacuum to obtain the title compounds $\mathbf{3} \mathbf{d}-\mathbf{3} \mathbf{j}$.

\subsubsection{General Procedure for Synthesis of Compounds $\mathbf{4 a - 6 j}$}

A mixture of intermediate compound $2(3.8 \mathrm{mmol})$ and $O$-substituted hydroxylamines intermediate compound $3(4.56 \mathrm{mmol})$ in ethyl alcohol $(10 \mathrm{~mL})$ was stirred at reflux for $1 \mathrm{~h}$. After the reaction was completed, the solvent was removed under reduced pressure, and purified by the column chromatography $\left(\mathrm{V}_{\text {petroleum ether }}: \mathrm{V}_{\text {ethyl acetate }}=1: 20\right)$ to produce title compounds $\mathbf{4 a - 6 j}$.

(E)-7-Hydroxy-2-oxo-4-(trifluoromethyl)-2H-chromene-8-carbaldehyde oxime (4a): white solid; mp 236.2-237.2 ${ }^{\circ} \mathrm{C}$; yield 60\%; ${ }^{1} \mathrm{H}-\mathrm{NMR}\left(400 \mathrm{MHz}, \mathrm{DMSO}-d_{6}\right) \delta 12.05(\mathrm{~s}, 1 \mathrm{H}), 11.46(\mathrm{~s}, 1 \mathrm{H})$, $8.55(\mathrm{t}, J=25.2 \mathrm{~Hz}, 1 \mathrm{H}), 7.60(\mathrm{~d}, J=6.8 \mathrm{~Hz}, 1 \mathrm{H}), 7.06(\mathrm{~d}, J=9.0 \mathrm{~Hz}, 1 \mathrm{H}), 6.89$ (d, J = 5.7 Hz, $1 \mathrm{H}) ;{ }^{13} \mathrm{C}-\mathrm{NMR}\left(101 \mathrm{MHz}, \mathrm{DMSO}-d_{6}\right) \delta 161.03,158.39,153.25,143.68,140.10(\mathrm{q}, J=32.0 \mathrm{~Hz})$, $126.70,122.10(\mathrm{~d}, J=275.6 \mathrm{~Hz}), 114.43,113.18(\mathrm{q}, J=6.3 \mathrm{~Hz}), 106.51,105.92 ; \mathrm{IR}(\mathrm{KBr}) \vee\left(\mathrm{cm}^{-1}\right)$ 3494, 3105, 3009, 2915, 1730, 1280; HR-MS (ESI): $m / z$ calcd. for $\mathrm{C}_{11} \mathrm{H}_{6} \mathrm{NF}_{3} \mathrm{O}_{4}\left([\mathrm{M}+\mathrm{H}]^{+}\right)$ 274.0249 , found 274.0490 .

(E)-7-hydroxy-2-oxo-4-(trifluoromethyl)-2H-chromene-8-carbaldehydeO-methyloxime (4b): white solid; mp 201.4-202.3 ${ }^{\circ} \mathrm{C}$, yield 34\%; ${ }^{1} \mathrm{H}-\mathrm{NMR}\left(400 \mathrm{MHz}, \mathrm{DMSO}-d_{6}\right) \delta 11.14(\mathrm{~s}, 1 \mathrm{H}), 8.50$ (s, $1 \mathrm{H}), 8.00-7.35(\mathrm{~m}, 1 \mathrm{H}), 7.07(\mathrm{~d}, J=9.0 \mathrm{~Hz}, 1 \mathrm{H}), 6.90(\mathrm{~s}, 1 \mathrm{H}), 3.99(\mathrm{~s}, 3 \mathrm{H}) ;{ }^{13} \mathrm{C}-\mathrm{NMR}(101 \mathrm{MHz}$, $\left.\mathrm{CDCl}_{3}\right) \delta 157.58,157.23,151.75,144.79,141.04(\mathrm{q}, J=33.7 \mathrm{~Hz}), 126.87,122.59,119.68(\mathrm{~d}$, $J=32.2 \mathrm{~Hz}), 113.09(\mathrm{q}, J=7.1 \mathrm{~Hz}), 106.22(\mathrm{~d}, J=9.4 \mathrm{~Hz}), 63.33,29.72 ; \mathrm{IR}(\mathrm{KBr}) \vee\left(\mathrm{cm}^{-1}\right)$ $3494,3105,3009,2956,1738,1284$; HR-MS (ESI): $m / z$ calcd. for $\mathrm{C}_{12} \mathrm{H}_{8} \mathrm{NF}_{3} \mathrm{O}_{4}\left([\mathrm{M}+\mathrm{H}]^{+}\right)$ 288.0405, found 288.0654 .

(E)-7-hydroxy-2-oxo-4-(trifluoromethyl)-2H-chromene-8-carbaldehyde O-ethyl oxime (4c): yellow solid; mp 199.8-199.9 ${ }^{\circ} \mathrm{C}$, yield 30\%; ${ }^{1} \mathrm{H}-\mathrm{NMR}\left(400 \mathrm{MHz}, \mathrm{CDCl}_{3}\right) \delta 11.15$ (s, 1H), 8.65 (s, $1 \mathrm{H}), 7.62(\mathrm{~d}, J=9.0 \mathrm{~Hz}, 1 \mathrm{H}), 7.01(\mathrm{~d}, J=9.1 \mathrm{~Hz}, 1 \mathrm{H}), 6.64(\mathrm{~s}, 1 \mathrm{H}), 1.82(\mathrm{dd}, J=14.2,7.0 \mathrm{~Hz}$, 2H), $1.02(\mathrm{t}, J=7.4 \mathrm{~Hz}, 3 \mathrm{H}) ;{ }^{13} \mathrm{C}-\mathrm{NMR}\left(101 \mathrm{MHz}, \mathrm{CDCl}_{3}\right) \delta 159.38,157.79,153.55,144.87$ $(\mathrm{q}, J=22.2 \mathrm{~Hz}), 142.07,123.89,118.40,112.67(\mathrm{q}, J=5.9 \mathrm{~Hz}), 105.91(\mathrm{~d}, J=49.6 \mathrm{~Hz}), 71.09$ $(\mathrm{d}, J=12.3 \mathrm{~Hz}), 70.23,14.57,14.23$; IR $(\mathrm{KBr}) \vee\left(\mathrm{cm}^{-1}\right) 3494,3100,2891,1744,1407,1288$; HR-MS (ESI): $m / z$ calcd. for $\mathrm{C}_{13} \mathrm{H}_{10} \mathrm{NF}_{3} \mathrm{O}_{4}\left([\mathrm{M}+\mathrm{H}]^{+}\right)$302.0562, found 302.0535 . 
(E)-7-hydroxy-2-oxo-4-(trifluoromethyl)-2H-chromene-8-carbaldehyde O-propyl oxime (4d): white solid; mp 169.9-170.0 ${ }^{\circ} \mathrm{C}$, yield 34\%; ${ }^{1} \mathrm{H}-\mathrm{NMR}\left(400 \mathrm{MHz}, \mathrm{CDCl}_{3}\right) \delta 11.15(\mathrm{~s}, 1 \mathrm{H}), 8.85(\mathrm{~s}, 1 \mathrm{H})$, $7.62(\mathrm{~d}, J=9.0 \mathrm{~Hz}, 1 \mathrm{H}), 6.99(\mathrm{~d}, J=9.1 \mathrm{~Hz}, 1 \mathrm{H}), 6.64(\mathrm{~s}, 1 \mathrm{H}), 4.20(\mathrm{t}, J=6.6 \mathrm{~Hz}, 2 \mathrm{H}), 1.79(\mathrm{dd}$, $J=14.2,7.0 \mathrm{~Hz}, 2 \mathrm{H}), 1.02(\mathrm{t}, J=7.4 \mathrm{~Hz}, 3 \mathrm{H}) ;{ }^{13} \mathrm{C}-\mathrm{NMR}\left(101 \mathrm{MHz}, \mathrm{CDCl}_{3}\right) \delta 161.81,158.32$, $153.48,144.91,141.85(\mathrm{q}, J=33.0 \mathrm{~Hz}), 127.23,122.85,120.11,114.75,111.79(\mathrm{q}, J=5.5 \mathrm{~Hz})$, 105.94, 105.48, 22.11, 10.25. IR (KBr) $v\left(\mathrm{~cm}^{-1}\right)$ 3092, 2983, 2941, 2884, 1741, 1286; HR-MS (ESI): $m / z$ calcd. for $\mathrm{C}_{14} \mathrm{H}_{12} \mathrm{NF}_{3} \mathrm{O}_{4}\left([\mathrm{M}+\mathrm{H}]^{+}\right)$316.0718, found 316.0689.

(E)-7-hydroxy-2-oxo-4-(trifluoromethyl)-2H-chromene-8-carbaldehyde O-butyl oxime (4e): white solid; mp 149.0-149.1 ${ }^{\circ} \mathrm{C}$; yield 51\%; ${ }^{1} \mathrm{H}-\mathrm{NMR}\left(400 \mathrm{MHz}, \mathrm{DMSO}-d_{6}\right) \delta 11.10(\mathrm{~s}, 1 \mathrm{H}), 8.49$ (s, $1 \mathrm{H}), 7.61(\mathrm{~d}, J=7.8 \mathrm{~Hz}, 1 \mathrm{H}), 7.04(\mathrm{~d}, J=9.0 \mathrm{~Hz}, 1 \mathrm{H}), 6.87(\mathrm{~s}, 1 \mathrm{H}), 4.21(\mathrm{t}, J=6.6 \mathrm{~Hz}, 2 \mathrm{H})$, $1.79-1.59(\mathrm{~m}, 2 \mathrm{H}), 1.54-1.29(\mathrm{~m}, 2 \mathrm{H}), 0.93(\mathrm{t}, J=7.4 \mathrm{~Hz}, 3 \mathrm{H}) ;{ }^{13} \mathrm{C}-\mathrm{NMR}\left(101 \mathrm{MHz}, \mathrm{DMSO}-d_{6}\right)$ $\delta 159.98,159.12,153.26,140.16(q, J=31.8 \mathrm{~Hz}), 133.41,123.60,123.50,120.86,118.08,114.11$, $113.46(\mathrm{q}, J=5.2 \mathrm{~Hz}), 109.84,107.02,69.45,8.49$; IR $(\mathrm{KBr}) \vee\left(\mathrm{cm}^{-1}\right) 3103,2941,2873,1743$, 1397, 1277; HR-MS (ESI): $m / z$ calcd. for $\mathrm{C}_{15} \mathrm{H}_{14} \mathrm{NF}_{3} \mathrm{O}_{4}\left([\mathrm{M}+\mathrm{H}]^{+}\right)$330.0875, found 330.1160 .

(E)-7-hydroxy-2-oxo-4-(trifluoromethyl)-2H-chromene-8-carbaldehyde O-allyl oxime (4f): white solid; mp 159.9-160.5 ${ }^{\circ} \mathrm{C}$; yield 76\%; ${ }^{1} \mathrm{H}-\mathrm{NMR}\left(500 \mathrm{MHz}, \mathrm{CDCl}_{3}\right) \delta 11.03$ (s), 11.03 (s), $8.86(\mathrm{~s}), 7.70-7.68(\mathrm{~m}), 7.60(\mathrm{dd}, J=9.1,1.5 \mathrm{~Hz}), 7.60(\mathrm{dd}, J=9.1,1.5 \mathrm{~Hz}), 6.96(\mathrm{~d}, J=9.1 \mathrm{~Hz})$, $6.62(\mathrm{~s}), 6.06-5.97(\mathrm{~m}), 5.42-5.36(\mathrm{~m}), 5.34-5.30(\mathrm{~m}), 4.69(\mathrm{dd}, J=6.0,0.8 \mathrm{~Hz}) .{ }^{13} \mathrm{C}-\mathrm{NMR}$ (101 MHz, $\left.\mathrm{CDCl}_{3}\right) \delta 161.82$ (s), 158.24 (s), 153.59 (s), 145.40 (s), 141.84 (q, J = 32.6 Hz), 132.83 (s), $129.85(\mathrm{~d}, J=208.9 \mathrm{~Hz}), 127.42(\mathrm{~d}, J=2.4 \mathrm{~Hz}), 119.50$ (s), 114.80 (s), 111.84 (q, J = 5.8 Hz), 105.95 (s), 105.36 (s), 76.09 (s). FTIR (KBr) v ( $\left.\mathrm{cm}^{-1}\right)$ 3450.6, 3092.1, 1736.2, 12292.2; HR-MS (ESI): $m / z$ calcd. for $\mathrm{C}_{14} \mathrm{H}_{10} \mathrm{~F}_{3} \mathrm{NO}_{4}\left([\mathrm{M}+\mathrm{H}]^{+}\right)$314.0635, found 314.0633.

(E)-7-hydroxy-2-oxo-4-(trifluoromethyl)-2H-chromene-8-carbaldehyde O-(3-methylbut-2-en-1-yl) oxime (4g): white solid; mp 165.9-166.4 ${ }^{\circ} \mathrm{C}$; yield 61\%; ${ }^{1} \mathrm{H}-\mathrm{NMR}\left(600 \mathrm{MHz}, \mathrm{CDCl}_{3}\right) \delta 11.16$ $(\mathrm{s}, 1 \mathrm{H}), 8.82(\mathrm{~s}, 1 \mathrm{H}), 7.60(\mathrm{dd}, J=9.1,1.8 \mathrm{~Hz}, 1 \mathrm{H}), 6.97(\mathrm{~d}, J=9.1 \mathrm{~Hz}, 1 \mathrm{H}), 6.62(\mathrm{~s}, 1 \mathrm{H})$, 5.50-5.44 (m, 1H), $4.71(\mathrm{~d}, J=7.3 \mathrm{~Hz}, 2 \mathrm{H}), 1.82(\mathrm{~s}, 3 \mathrm{H}), 1.79(\mathrm{~d}, J=0.7 \mathrm{~Hz}, 3 \mathrm{H}) .{ }^{13} \mathrm{C}-\mathrm{NMR}$ $\left(151 \mathrm{MHz}, \mathrm{CDCl}_{3}\right) \delta 161.87$ (s), 158.28 (s), 153.54 (s), 145.02 (s), 141.84 (q, J = 32.8 Hz), $140.23(\mathrm{~s}), 127.23(\mathrm{~d}, J=2.3 \mathrm{~Hz}), 121.52(\mathrm{~d}, J=275.7 \mathrm{~Hz}), 118.81(\mathrm{~s}), 114.75$ (s), 111.81 (q, $J=5.8 \mathrm{~Hz}), 105.93$ (s), $105.58(\mathrm{~s}), 71.65$ (s), 25.86 (s), 18.16 (s). FTIR (KBr) v (cm $\left.{ }^{-1}\right) 3476.1$, 3104.2, 1753.1, 1294, 1278.7; HR-MS (ESI): $m / z$ calcd. for $\mathrm{C}_{16} \mathrm{H}_{14} \mathrm{~F}_{3} \mathrm{NO}_{4}\left([\mathrm{M}+\mathrm{H}]^{+}\right) 342.0948$, found 342.0945 .

(E)-7-hydroxy-2-oxo-4-(trifluoromethyl)-2H-chromene-8-carbaldehyde O-prop-2-yn-1-yl oxime (4h): white solid; mp 189.9-190.2 ${ }^{\circ} \mathrm{C}$; yield 56\%; ${ }^{1} \mathrm{H}-\mathrm{NMR}\left(600 \mathrm{MHz}, \mathrm{CDCl}_{3}\right) \delta 10.86$ (s, $1 \mathrm{H}), 8.90(\mathrm{~s}, 1 \mathrm{H}), 7.65(\mathrm{dd}, J=9.1,1.8 \mathrm{~Hz}, 1 \mathrm{H}), 7.01(\mathrm{~d}, J=9.1 \mathrm{~Hz}, 1 \mathrm{H}), 6.64(\mathrm{~s}, 1 \mathrm{H}), 4.81(\mathrm{~d}$, $J=2.4 \mathrm{~Hz}, 2 \mathrm{H}), 2.57(\mathrm{t}, J=2.4 \mathrm{~Hz}, 1 \mathrm{H}) .{ }^{13} \mathrm{C}-\mathrm{NMR}\left(151 \mathrm{MHz}, \mathrm{CDCl}_{3}\right) \delta 161.85(\mathrm{~s}), 158.15(\mathrm{~s})$, $153.80(\mathrm{~s}), 146.57$ (s), $141.84(\mathrm{q}, J=32.8 \mathrm{~Hz}), 127.85$ (d, $J=2.4 \mathrm{~Hz}), 121.47$ (q, J = 275.6 Hz), 114.91 (s), 111.95 (q, J = 5.8 Hz), 106.03 (s), 105.09 (s), 78.00 (s), 76.01 (s), 62.57 (s). FTIR (KBr) $v\left(\mathrm{~cm}^{-1}\right)$ 3476.6, 3110.8, 2962.9, 1737.4, 1401.5; HR-MS (ESI): $m / z$ calcd. for $\mathrm{C}_{14} \mathrm{H}_{8} \mathrm{~F}_{3} \mathrm{NO}_{4}$ $\left([\mathrm{M}+\mathrm{H}]^{+}\right) 312.0478$, found 312.0473 .

(E)-7-hydroxy-2-oxo-4-(trifluoromethyl)-2H-chromene-8-carbaldehyde O-benzyl oxime (4i): white solid; mp 169.5-170.4 ${ }^{\circ} \mathrm{C}$; yield 75\%; ${ }^{1} \mathrm{H}-\mathrm{NMR}\left(600 \mathrm{MHz}, \mathrm{CDCl}_{3}\right) \delta 10.93(\mathrm{~s}, 1 \mathrm{H}), 8.89$ (s, $1 \mathrm{H}), 7.60(\mathrm{dd}, J=9.1,1.7 \mathrm{~Hz}, 1 \mathrm{H}), 7.41(\mathrm{~s}, 1 \mathrm{H}), 7.40(\mathrm{~d}, J=2.2 \mathrm{~Hz}, 2 \mathrm{H}), 7.38-7.34(\mathrm{~m}, 1 \mathrm{H})$, $6.95(\mathrm{~d}, J=9.1 \mathrm{~Hz}, 1 \mathrm{H}), 6.62(\mathrm{~s}, 1 \mathrm{H}), 5.23(\mathrm{~s}, 2 \mathrm{H}) .{ }^{13} \mathrm{C}-\mathrm{NMR}\left(151 \mathrm{MHz}, \mathrm{CDCl}_{3}\right) \delta 161.79$ (s), 158.20 (s), 153.60 (s), 145.76 (s), 141.82 (q, J = 32.9 Hz), 136.14 (s), 128.73 (s), 128.59 (s), 128.57 (s), $127.45(\mathrm{~d}, J=2.3 \mathrm{~Hz}), 121.48(\mathrm{q}, J=275.5 \mathrm{~Hz}), 114.77(\mathrm{~s}), 111.85$ (q, J = 5.7 Hz), 105.95 (s), 105.35 (s), 77.30 (s). FTIR (KBr) $v\left(\mathrm{~cm}^{-1}\right)$ 3494, 3105, 3009, 2915, 1730, 1280; HR-MS (ESI): $m / z$ calcd. for $\mathrm{C}_{18} \mathrm{H}_{12} \mathrm{~F}_{3} \mathrm{NO}_{4}\left([\mathrm{M}+\mathrm{H}]^{+}\right)$364.0791, found 364.0795 .

(E)-7-hydroxy-2-oxo-4-(trifluoromethyl)-2H-chromene-8-carbaldehyde O-(4-fluorobenzyl) oxime (4j): white solid; mp 175.7-176.2 ${ }^{\circ} \mathrm{C}$; yield 74\%; ${ }^{1} \mathrm{H}-\mathrm{NMR}\left(600 \mathrm{MHz}, \mathrm{CDCl}_{3}\right) \delta 10.88$ (s, $1 \mathrm{H}), 8.88(\mathrm{~s}, 1 \mathrm{H}), 7.61(\mathrm{dd}, J=9.1,1.8 \mathrm{~Hz}, 1 \mathrm{H}), 7.40-7.38(\mathrm{~m}, 2 \mathrm{H}), 7.09(\mathrm{~d}, J=8.7 \mathrm{~Hz}, 2 \mathrm{H})$, $6.96(\mathrm{~d}, J=9.0 \mathrm{~Hz}, 1 \mathrm{H}), 6.62(\mathrm{~s}, 1 \mathrm{H}), 5.19(\mathrm{~s}, 2 \mathrm{H}) .{ }^{13} \mathrm{C}-\mathrm{NMR}\left(151 \mathrm{MHz}, \mathrm{CDCl}_{3}\right) \delta 162.88(\mathrm{~d}$, $J=247.3 \mathrm{~Hz}$ ), 161.77 (s), 158.18 (s), 153.63 (s), 145.98 (s), 141.83 (q, J = 32.8 Hz), 131.98 (d, 
$J=3.3 \mathrm{~Hz}), 130.46(\mathrm{~d}, J=8.3 \mathrm{~Hz}), 127.57(\mathrm{~d}, J=2.3 \mathrm{~Hz}), 121.47(\mathrm{q}, J=275.6 \mathrm{~Hz}), 115.71(\mathrm{~d}$, $J=21.6 \mathrm{~Hz}), 114.78(\mathrm{~s}), 111.89(\mathrm{q}, J=5.7 \mathrm{~Hz}), 106.00(\mathrm{~s}), 105.27(\mathrm{~s}), 76.49(\mathrm{~s})$. FTIR $(\mathrm{KBr}) v$ $\left(\mathrm{cm}^{-1}\right)$ 3476.9, 3105.7, 1752.2, 1293.5, 1277.7; HR-MS (ESI): $\mathrm{m} / z$ calcd. for $\mathrm{C}_{18} \mathrm{H}_{11} \mathrm{~F}_{4} \mathrm{NO}_{4}$ $\left([\mathrm{M}+\mathrm{H}]^{+}\right)$382.0697, found 382.0694.

(E)-6-chloro-7-hydroxy-2-oxo-4-(trifluoromethyl)-2H-chromene-8-carbaldehyde oxime (5a): yellow solid; mp 121.5-122.0 ${ }^{\circ} \mathrm{C}$; yield 70\%; ${ }^{1} \mathrm{H}-\mathrm{NMR}\left(400 \mathrm{MHz}\right.$, DMSO- $\left.d_{6}\right) \delta 12.47(\mathrm{~s}, 1 \mathrm{H}), 12.21$ $(\mathrm{s}, 1 \mathrm{H}), 8.60(\mathrm{~s}, 1 \mathrm{H}), 7.65(\mathrm{~s}, 1 \mathrm{H}), 7.04(\mathrm{~s}, 1 \mathrm{H}) ;{ }^{13} \mathrm{C}-\mathrm{NMR}\left(101 \mathrm{MHz}, \mathrm{DMSO}-d_{6}\right) \delta 157.67$, $156.31,151.54,143.97,139.03(\mathrm{q}, J=32.9 \mathrm{~Hz}), 125.35,121.80(\mathrm{~d}, J=275.8 \mathrm{~Hz}), 117.96,114.92$ $(\mathrm{q}, J=5.7 \mathrm{~Hz}), 107.18,106.40$; IR $(\mathrm{KBr}) \vee\left(\mathrm{cm}^{-1}\right) 3435,3086,3006,2879,1725,1279$; HR-MS (ESI): $m / z$ calcd. for $\mathrm{C}_{11} \mathrm{H}_{4} \mathrm{ClF}_{3} \mathrm{O}_{4}\left([\mathrm{M}+\mathrm{H}]^{+}\right) 307.9859$, found 307.9824 .

(E)-6-chloro-7-hydroxy-2-oxo-4-(trifluoromethyl)-2H-chromene-8-carbaldehyde O-methyl oxime (5b): white solid; mp 203.8-204.5 ${ }^{\circ} \mathrm{C}$; yield 55\%; ${ }^{1} \mathrm{H}-\mathrm{NMR}\left(400 \mathrm{MHz}\right.$, DMSO- $\left.d_{6}\right) \delta 11.47$ (s, 1H), $8.62(\mathrm{~s}, 1 \mathrm{H}), 7.70(\mathrm{~s}, 1 \mathrm{H}), 7.05(\mathrm{~s}, 1 \mathrm{H}), 4.06(\mathrm{~s}, 3 \mathrm{H}) ;{ }^{13} \mathrm{C}-\mathrm{NMR}\left(101 \mathrm{MHz}, \mathrm{CDCl}_{3}\right) \delta 157.58$, $157.23,151.75,144.79,141.04(\mathrm{q}, J=33.7 \mathrm{~Hz}), 126.87,122.59,119.68(\mathrm{~d}, J=32.2 \mathrm{~Hz}), 113.09(\mathrm{q}$, $J=6.0 \mathrm{~Hz}), 106.22(\mathrm{~d}, J=9.4 \mathrm{~Hz}), 63.33,29.72 ; \mathrm{IR}(\mathrm{KBr}) \vee\left(\mathrm{cm}^{-1}\right) 3434,3085,2918,1725,1399$, 1279; HR-MS (ESI): $m / z$ calcd. for $\mathrm{C}_{12} \mathrm{H}_{7} \mathrm{ClF}_{3} \mathrm{NO}_{4}\left([\mathrm{M}+\mathrm{Na}]^{+}\right) 322.0016$, found 321.9968 .

(E)-6-chloro-7-hydroxy-2-oxo-4-(trifluoromethyl)-2H-chromene-8-carbaldehyde O-ethyl oxime (5c): white solid; mp 194.7-194.8 ${ }^{\circ} \mathrm{C}$; yield $46 \%$; ${ }^{1} \mathrm{H}-\mathrm{NMR}\left(400 \mathrm{MHz}, \mathrm{DMSO}-d_{6}\right) \delta 11.53(\mathrm{~s}, 1 \mathrm{H})$, $8.62(\mathrm{~s}, 1 \mathrm{H}), 7.68(\mathrm{~s}, 1 \mathrm{H}), 7.05(\mathrm{~s}, 1 \mathrm{H}), 4.34(\mathrm{dd}, J=14.0,7.0 \mathrm{~Hz}, 2 \mathrm{H}), 1.31(\mathrm{t}, J=7.0 \mathrm{~Hz}, 3 \mathrm{H})$; ${ }^{13} \mathrm{C}-\mathrm{NMR}\left(101 \mathrm{MHz}, \mathrm{CDCl}_{3}\right) \delta 157.57,157.22,151.71,144.57,141.19,140.86(\mathrm{q}, J=32.0 \mathrm{~Hz})$, $126.71,119.46,113.07(\mathrm{q}, J=5.7 \mathrm{~Hz}), 106.39,106.23,71.40,14.20 ; \mathrm{IR}(\mathrm{KBr}) \vee\left(\mathrm{cm}^{-1}\right) 3209,3086$, 2901, 1731, 1400, 1278; HR-MS (ESI): $m / z$ calcd. for $\mathrm{C}_{13} \mathrm{H}_{9} \mathrm{ClF}_{3} \mathrm{NO}_{4}\left([\mathrm{M}+\mathrm{H}]^{+}\right) 336.0172$, found 336.0123 .

(E)-6-chloro-7-hydroxy-2-oxo-4-(trifluoromethyl)-2H-chromene-8-carbaldehyde O-propyl oxime (5d): white solid; mp 179.9-180.9 ${ }^{\circ} \mathrm{C}$; yield $40 \%$; ${ }^{1} \mathrm{H}-\mathrm{NMR}\left(400 \mathrm{MHz}\right.$, DMSO- $\left.d_{6}\right) \delta 11.54$ (s, $1 \mathrm{H}), 8.63(\mathrm{~s}, 1 \mathrm{H}), 7.68(\mathrm{~s}, 1 \mathrm{H}), 7.05(\mathrm{~s}, 1 \mathrm{H}), 4.25(\mathrm{t}, J=6.6 \mathrm{~Hz}, 2 \mathrm{H}), 1.71(\mathrm{dt}, J=14.1,7.0 \mathrm{~Hz}$, 2H), $0.96(\mathrm{t}, J=7.4 \mathrm{~Hz}, 3 \mathrm{H}) ;{ }^{13} \mathrm{C}-\mathrm{NMR}\left(101 \mathrm{MHz}, \mathrm{CDCl}_{3}\right) \delta 157.59,157.21,151.69,144.48$, $141.63(\mathrm{q}, J=32.0 \mathrm{~Hz}), 126.66,122.60,119.86,119.46,113.05(\mathrm{q}, J=5.6 \mathrm{~Hz}), 106.41,106.23$, 22.08, 10.20; IR (KBr) $v\left(\mathrm{~cm}^{-1}\right) 3208,3083,2963,1730,1399$, 1290; HR-MS (ESI): $m / z$ calcd. for $\mathrm{C}_{14} \mathrm{H}_{11} \mathrm{ClF}_{3} \mathrm{NO}_{4}\left([\mathrm{M}+\mathrm{H}]^{+}\right)$350.0329, found 350.0637.

(E)-6-chloro-7-hydroxy-2-oxo-4-(trifluoromethyl)-2H-chromene-8-carbaldehyde O-butyl oxime (5e): white solid; mp 164.9-165.6 ${ }^{\circ} \mathrm{C}$; yield $42 \%$; ${ }^{1} \mathrm{H}-\mathrm{NMR}\left(400 \mathrm{MHz}, \mathrm{DMSO}-d_{6}\right) \delta 11.54(\mathrm{~s}, 1 \mathrm{H})$, $8.62(\mathrm{~s}, 1 \mathrm{H}), 7.67(\mathrm{~s}, 1 \mathrm{H}), 7.05(\mathrm{~s}, 1 \mathrm{H}), 4.29(\mathrm{t}, J=6.6 \mathrm{~Hz}, 2 \mathrm{H}), 1.75-1.64(\mathrm{~m}, 2 \mathrm{H}), 1.41(\mathrm{dq}$, $J=14.7,7.4 \mathrm{~Hz}, 2 \mathrm{H}), 0.94(\mathrm{t}, J=7.4 \mathrm{~Hz}, 3 \mathrm{H}) ;{ }^{13} \mathrm{C}-\mathrm{NMR}\left(151 \mathrm{MHz}, \mathrm{CDCl}_{3}\right) \delta 157.60$ (s), 157.26 (s), $151.73(\mathrm{~s}), 144.48(\mathrm{~s}), 141.08(\mathrm{q}, J=33.2 \mathrm{~Hz}), 126.69(\mathrm{~d}, J=2.4 \mathrm{~Hz}), 121.25(\mathrm{q}, J=275.8 \mathrm{~Hz})$, 119.49 (s), $113.06(\mathrm{q}, J=5.7 \mathrm{~Hz}), 106.45(\mathrm{~s}), 106.25(\mathrm{~s}), 75.67$ (s), $30.78(\mathrm{~s}), 18.99$ (s), $13.80(\mathrm{~s})$. $\mathrm{IR}(\mathrm{KBr}) \vee\left(\mathrm{cm}^{-1}\right) 3082,2966,2936,1744,1290$; HR-MS (ESI): $m / z$ calcd. for $\mathrm{C}_{15} \mathrm{H}_{13} \mathrm{ClF}_{3} \mathrm{NO}_{4}$ $\left([\mathrm{M}+\mathrm{H}]^{+}\right)$364.0485, found 364.0815.

(E)-6-chloro-7-hydroxy-2-oxo-4-(trifluoromethyl)-2H-chromene-8-carbaldehyde O-allyl oxime (5f): white solid; mp 169.5-169.9 ${ }^{\circ} \mathrm{C}$; yield 83\%; ${ }^{1} \mathrm{H}-\mathrm{NMR}\left(400 \mathrm{MHz}, \mathrm{CDCl}_{3}\right) \delta 11.73(\mathrm{~s}, 1 \mathrm{H})$, $8.85(\mathrm{~s}, 1 \mathrm{H}), 7.72(\mathrm{~d}, J=1.5 \mathrm{~Hz}, 1 \mathrm{H}), 6.69(\mathrm{~s}, 1 \mathrm{H}), 6.04(\mathrm{~m}, J=16.5,10.4,6.1 \mathrm{~Hz}, 1 \mathrm{H}), 5.44$ $(\mathrm{dd}, J=17.2,1.4 \mathrm{~Hz}, 1 \mathrm{H}), 5.38(\mathrm{dd}, J=10.4,1.1 \mathrm{~Hz}, 1 \mathrm{H}), 4.74(\mathrm{~d}, J=6.0 \mathrm{~Hz}, 2 \mathrm{H}) .{ }^{13} \mathrm{C}-\mathrm{NMR}$ $\left(101 \mathrm{MHz}, \mathrm{CDCl}_{3}\right) \delta 157.55$ (s), 157.21 (s), 151.77 (s), 144.95 (s), 142.39-139.57 (m), 132.50 (s), $126.83(\mathrm{~d}, J=2.6 \mathrm{~Hz}), 122.58(\mathrm{~s}), 120.00(\mathrm{~s}), 119.50(\mathrm{~s}), 113.08(\mathrm{q}, J=5.6 \mathrm{~Hz}), 106.28(\mathrm{~s}), 106.23$ (s), 76.38 (s). FTIR (KBr) $v\left(\mathrm{~cm}^{-1}\right) 3570.1,1744.7,1640.7,1402.0 ;$ HR-MS (ESI): $m / z$ calcd. for $\mathrm{C}_{14} \mathrm{H}_{9} \mathrm{ClF}_{3} \mathrm{NO}_{4}\left([\mathrm{M}+\mathrm{H}]^{+}\right)$348.0245, found 348.0247.

(E)-6-chloro-7-hydroxy-2-oxo-4-(trifluoromethyl)-2H-chromene-8-carbaldehyde O-(3-methylbut-2en-1-yl) oxime (5g): white solid; mp 163.4-164.2 ${ }^{\circ} \mathrm{C}$; yield $72 \% ;{ }^{1} \mathrm{H}-\mathrm{NMR}\left(600 \mathrm{MHz}, \mathrm{CDCl}_{3}\right) \delta$ $11.84(\mathrm{~s}, 1 \mathrm{H}), 8.80(\mathrm{~s}, 1 \mathrm{H}), 7.70(\mathrm{~s}, 1 \mathrm{H}), 6.67(\mathrm{~s}, 1 \mathrm{H}), 5.47(\mathrm{t}, J=7.8 \mathrm{~Hz}, 1 \mathrm{H}), 4.73(\mathrm{~d}, J=7.3 \mathrm{~Hz}$, 2H), $1.82(\mathrm{~s}, 3 \mathrm{H}), 1.79(\mathrm{~s}, 3 \mathrm{H}) .{ }^{13} \mathrm{C}-\mathrm{NMR}\left(151 \mathrm{MHz}, \mathrm{CDCl}_{3}\right) \delta 157.61(\mathrm{~s}), 157.31(\mathrm{~s}), 151.74(\mathrm{~s})$, $144.62(\mathrm{~s}), 141.06(\mathrm{q}, J=33.1 \mathrm{~Hz}), 140.63(\mathrm{~s}), 126.67(\mathrm{~d}, J=2.3 \mathrm{~Hz}), 121.26(\mathrm{~d}, J=275.6 \mathrm{~Hz})$, 
119.48 (s), 118.53 (s), 113.04 (q, J = 5.7 Hz), 106.51 (s), 106.21 (s), 71.97 (s), 25.87 (s), 18.21 (s). FTIR $(\mathrm{KBr}) v\left(\mathrm{~cm}^{-1}\right)$ 3476.6, 3476.6, 3414.2, 1744.5, 1288.6; HR-MS (ESI): $\mathrm{m} / z$ calcd. for $\mathrm{C}_{16} \mathrm{H}_{13} \mathrm{ClF}_{3} \mathrm{NO}_{4}\left([\mathrm{M}+\mathrm{H}]^{+}\right)$376.0558, found 376.0556 .

(E)-6-chloro-7-hydroxy-2-oxo-4-(trifluoromethyl)-2H-chromene-8-carbaldehyde O-prop-2-yn-1-yl oxime (5h): white solid; mp 179.9-180.4 ${ }^{\circ} \mathrm{C}$; yield 54\%; ${ }^{1} \mathrm{H}-\mathrm{NMR}\left(600 \mathrm{MHz}, \mathrm{CDCl}_{3}\right) \delta 11.53$ $(\mathrm{s}, 1 \mathrm{H}), 8.88(\mathrm{~s}, 1 \mathrm{H}), 7.75(\mathrm{~d}, J=1.4 \mathrm{~Hz}, 1 \mathrm{H}), 6.69(\mathrm{~s}, 1 \mathrm{H}), 4.82(\mathrm{~d}, J=2.4 \mathrm{~Hz}, 2 \mathrm{H}), 2.59(\mathrm{t}$, $J=2.4 \mathrm{~Hz}, 1 \mathrm{H}) .{ }^{13} \mathrm{C}-\mathrm{NMR}\left(151 \mathrm{MHz}, \mathrm{CDCl}_{3}\right) \delta 157.46$ (s), 157.28 (s), 151.99 (s), 146.13 (s), $141.04(\mathrm{q}, J=33.2 \mathrm{~Hz}), 127.29(\mathrm{~d}, J=2.4 \mathrm{~Hz}), 121.21(\mathrm{~d}, J=275.6 \mathrm{~Hz}), 119.68(\mathrm{~s}), 113.18$ $(\mathrm{q}, J=5.7 \mathrm{~Hz}), 106.33(\mathrm{~s}), 106.04(\mathrm{~s}), 77.65$ (s), 76.40 (s), 62.81 (s). FTIR (KBr) $v\left(\mathrm{~cm}^{-1}\right)$ 3552.7, 3464.2, 3091.2, 2131.6, 1736.3, 1286.8; HR-MS (ESI): $m / z$ calcd. for $\mathrm{C}_{14} \mathrm{H}_{7} \mathrm{ClF}_{3} \mathrm{NO}_{4}$ $\left([\mathrm{M}+\mathrm{H}]^{+}\right) 346.0088$, found 346.0086 .

(E)-6-chloro-7-hydroxy-2-oxo-4-(trifluoromethyl)-2H-chromene-8-carbaldehyde O-benzyl oxime (5i): white solid; mp 185.4-185.9 ${ }^{\circ} \mathrm{C}$; yield 56\%; ${ }^{1} \mathrm{H}-\mathrm{NMR}\left(600 \mathrm{MHz}, \mathrm{CDCl}_{3}\right) \delta 11.60$ (s, $1 \mathrm{H}), 8.87(\mathrm{~s}, 1 \mathrm{H}), 7.70(\mathrm{~d}, J=1.3 \mathrm{~Hz}, 1 \mathrm{H}), 7.41(\mathrm{~s}, 2 \mathrm{H}), 7.41(\mathrm{~s}, 2 \mathrm{H}), 7.38(\mathrm{dd}, J=8.2,4.3 \mathrm{~Hz}$, 1H), 6.67 (s, 1H), 5.24 (s, 2H). ${ }^{13} \mathrm{C}-\mathrm{NMR}\left(151 \mathrm{MHz}, \mathrm{CDCl}_{3}\right) \delta 157.52$ (s), 157.23 (s), 151.80 (s), 145.39 (s), $141.02(\mathrm{q}, J=33.3 \mathrm{~Hz}), 135.80$ (s), 128.81 (s), 128.73 (s), 128.59 (s), 126.90 (d, $J=2.4 \mathrm{~Hz}), 121.21(\mathrm{q}, J=275.8 \mathrm{~Hz}), 119.50(\mathrm{~s}), 113.08(\mathrm{q}, J=5.7 \mathrm{~Hz}), 106.29$ (s), 106.24 (s), 77.61 (s). FTIR $(\mathrm{KBr}) \vee\left(\mathrm{cm}^{-1}\right) 3452.2,3085.8,1753.1,1401.7,1287.7 ;$ HR-MS (ESI): $\mathrm{m} / z$ calcd. for $\mathrm{C}_{18} \mathrm{H}_{11} \mathrm{ClF}_{3} \mathrm{NO}_{4}\left([\mathrm{M}+\mathrm{H}]^{+}\right)$398.0401, found 398.0397.

(E)-6-chloro-7-hydroxy-2-oxo-4-(trifluoromethyl)-2H-chromene-8-carbaldehyde O-(4-fluorobenzyl) oxime (5j): white solid; mp 200.7-201.2 ${ }^{\circ} \mathrm{C}$; yield 71\%; ${ }^{1} \mathrm{H}-\mathrm{NMR}\left(600 \mathrm{MHz}, \mathrm{CDCl}_{3}\right) \delta 11.54$ $(\mathrm{s}, 1 \mathrm{H}), 8.86(\mathrm{~s}, 1 \mathrm{H}), 7.70(\mathrm{~d}, J=1.4 \mathrm{~Hz}, 1 \mathrm{H}), 7.40-7.38(\mathrm{~m}, 2 \mathrm{H}), 7.10(\mathrm{t}, J=8.7 \mathrm{~Hz}, 2 \mathrm{H}), 6.67(\mathrm{~s}$, 1H), 5.20 (s, 2H). ${ }^{13} \mathrm{C}-\mathrm{NMR}\left(151 \mathrm{MHz}, \mathrm{CDCl}_{3}\right) \delta 162.88$ (d, J = 247.3 Hz), 161.77 (s), 158.18 (s), $153.63(\mathrm{~s}), 145.98(\mathrm{~s}), 141.83(\mathrm{q}, J=32.8 \mathrm{~Hz}), 131.98(\mathrm{~d}, J=3.3 \mathrm{~Hz}), 130.46(\mathrm{~d}, J=8.3 \mathrm{~Hz})$, $127.57(\mathrm{~d}, J=2.3 \mathrm{~Hz}), 121.47(\mathrm{q}, J=275.6 \mathrm{~Hz}), 115.71(\mathrm{~d}, J=21.6 \mathrm{~Hz}), 114.78$ (s), 111.89 $(\mathrm{q}, J=5.7 \mathrm{~Hz}), 106.00(\mathrm{~s}), 105.27(\mathrm{~s}), 76.49(\mathrm{~s})$. FTIR $(\mathrm{KBr}) \vee\left(\mathrm{cm}^{-1}\right)$ 3476.7, 3414.7, 3085.4, 1752.9, 1401.5, 1212.2; HR-MS (ESI): $m$ / $z$ calcd. for $\mathrm{C}_{18} \mathrm{H}_{10} \mathrm{ClF}_{4} \mathrm{NO}_{4}\left([\mathrm{M}+\mathrm{H}]^{+}\right) 416.0307$, found 416.0304 .

(E)-6-chloro-3-fluoro-7-hydroxy-4-methyl-2-oxo-2H-chromene-8-carbaldehyde oxime (6a): white solid; mp 240.1-241.6 ${ }^{\circ} \mathrm{C}$; yield 24\%; ${ }^{1} \mathrm{H}-\mathrm{NMR}\left(400 \mathrm{MHz}, \mathrm{DMSO}-d_{6}\right) \delta 12.35(\mathrm{~s}, 1 \mathrm{H}), 11.82$ $(\mathrm{s}, 1 \mathrm{H}), 8.58(\mathrm{~s}, 1 \mathrm{H}), 7.91(\mathrm{~s}, 1 \mathrm{H}), 2.37(\mathrm{~d}, J=2.4 \mathrm{~Hz}, 3 \mathrm{H}) ;{ }^{13} \mathrm{C}-\mathrm{NMR}\left(101 \mathrm{MHz}, \mathrm{CDCl}_{3}\right)$ $\delta 155.15(\mathrm{~d}, J=2.4 \mathrm{~Hz}), 153.76(\mathrm{~d}, J=30.5 \mathrm{~Hz}), 147.23,144.96,143.87,141.38,130.73(\mathrm{~d}$, $J=13.6 \mathrm{~Hz}), 126.29(\mathrm{~d}, J=6.0 \mathrm{~Hz}), 119.15,112.16,105.73,63.24,10.34(\mathrm{~d}, J=3.5 \mathrm{~Hz}) ; \mathrm{IR}(\mathrm{KBr})$ $v\left(\mathrm{~cm}^{-1}\right)$ 3381.78, 3077.75, 2921.54, 2850.53, 1703.49, 1320.36; HR-MS (ESI): $\mathrm{m} / z$ calcd. for $\mathrm{C}_{11} \mathrm{H}_{7} \mathrm{ClFNO}_{4}\left([\mathrm{M}+\mathrm{Na}]^{+}\right)$294.0048, found 294.0133 .

(E)-6-chloro-3-fluoro-7-hydroxy-4-methyl-2-oxo-2H-chromene-8-carbaldehyde O-methyl oxime (6b): white solid; mp 184.9-186.2 ${ }^{\circ} \mathrm{C}$; yield 33\%; ${ }^{1} \mathrm{H}-\mathrm{NMR}\left(400 \mathrm{MHz}, \mathrm{CDCl}_{3}\right) \delta 11.47$ $(\mathrm{s}, 1 \mathrm{H}), 8.82(\mathrm{~s}, 1 \mathrm{H}), 7.64-7.54(\mathrm{~m}, 1 \mathrm{H}), 4.08(\mathrm{~s}, 3 \mathrm{H}), 2.40(\mathrm{~d}, J=2.7 \mathrm{~Hz}, 3 \mathrm{H}) ;{ }^{13} \mathrm{C}-\mathrm{NMR}$ $\left(101 \mathrm{MHz}, \mathrm{CDCl}_{3}\right) \delta 155.15(\mathrm{~d}, J=2.4 \mathrm{~Hz}), 153.76(\mathrm{~d}, J=30.5 \mathrm{~Hz}), 147.23,144.96,142.62$ $(\mathrm{d}, J=250.0 \mathrm{~Hz}), 130.73(\mathrm{~d}, J=13.6 \mathrm{~Hz}), 126.29(\mathrm{~d}, J=6.0 \mathrm{~Hz}), 119.15,112.16,105.73,63.24$, $10.34(\mathrm{~d}, J=3.5 \mathrm{~Hz})$; IR (KBr) $v\left(\mathrm{~cm}^{-1}\right)$ 3489.32, 2955.37, 2923.50, 1738.95, 1306.48; HR-MS (ESI): $m / z$ calcd. for $\mathrm{C}_{12} \mathrm{H}_{9} \mathrm{ClFNO}_{4}\left([\mathrm{M}+\mathrm{Na}]^{+}\right)$308.0204, found 308.0311.

(E)-6-chloro-3-fluoro-7-hydroxy-4-methyl-2-oxo-2H-chromene-8-carbaldehyde O-ethyl oxime (6c): white solid; mp 163.9-164.1 ${ }^{\circ} \mathrm{C}$; yield 36\%; ${ }^{1} \mathrm{H}-\mathrm{NMR}\left(400 \mathrm{MHz}, \mathrm{CDCl}_{3}\right) \delta 11.60(\mathrm{~s}, 1 \mathrm{H})$, $8.84(\mathrm{~s}, 1 \mathrm{H}), 7.58(\mathrm{~s}, 1 \mathrm{H}), 4.32(\mathrm{q}, J=7.1 \mathrm{~Hz}, 2 \mathrm{H}), 2.40(\mathrm{~d}, J=2.8 \mathrm{~Hz}, 3 \mathrm{H}), 1.40(\mathrm{t}, J=7.1 \mathrm{~Hz}$, $3 \mathrm{H}) ;{ }^{13} \mathrm{C}-\mathrm{NMR}\left(101 \mathrm{MHz}, \mathrm{CDCl}_{3}\right) \delta 155.13(\mathrm{~d}, J=2.4 \mathrm{~Hz}), 154.62(\mathrm{~d}, J=30.1 \mathrm{~Hz}), 147.21$, 144.76, $141.75(\mathrm{~d}, J=250.0 \mathrm{~Hz}), 130.64(\mathrm{~d}, J=13.5 \mathrm{~Hz}), 126.14(\mathrm{~d}, J=6.0 \mathrm{~Hz}), 119.10$, $112.14,105.97,71.25,14.19,10.32(\mathrm{~d}, J=3.5 \mathrm{~Hz})$; IR $(\mathrm{KBr}) \vee\left(\mathrm{cm}^{-1}\right) 3489.52,2981.09,2956.24$, 2923.25, 1748.64, 1288.04; HR-MS (ESI): $m / z$ calcd. for $\mathrm{C}_{13} \mathrm{H}_{11} \mathrm{ClFNO}_{4}\left([\mathrm{M}+\mathrm{Na}]^{+}\right) 322.0361$, found 322.0466 . 
(E)-6-chloro-3-fluoro-7-hydroxy-4-methyl-2-oxo-2H-chromene-8-carbaldehyde O-propyl oxime (6d): yellow solid; mp 136.8-139.2 ${ }^{\circ} \mathrm{C}$; yield 27\%; ${ }^{1} \mathrm{H}-\mathrm{NMR}\left(400 \mathrm{MHz}, \mathrm{CDCl}_{3}\right) \delta 11.59(\mathrm{~s}, 1 \mathrm{H})$, $8.84(\mathrm{~s}, 1 \mathrm{H}), 7.57(\mathrm{~s}, 1 \mathrm{H}), 4.22(\mathrm{t}, J=6.6 \mathrm{~Hz}, 2 \mathrm{H}), 2.40(\mathrm{~d}, J=2.7 \mathrm{~Hz}, 3 \mathrm{H}), 1.79(\mathrm{dt}, J=14.1,7.0$ $\mathrm{Hz}, 2 \mathrm{H}), 1.02(\mathrm{t}, J=7.4 \mathrm{~Hz}, 3 \mathrm{H}) ;{ }^{13} \mathrm{C}-\mathrm{NMR}\left(101 \mathrm{MHz}, \mathrm{CDCl}_{3}\right) \delta 155.19(\mathrm{~d}, J=2.3 \mathrm{~Hz}), 153.96$ $(\mathrm{d}, J=4.4 \mathrm{~Hz}), 153.68,147.24(\mathrm{~d}, J=2.5 \mathrm{~Hz}), 144.74,143.87,130.79,126.13(\mathrm{~d}, J=6.0 \mathrm{~Hz})$, $119.13,112.17,106.04,22.09,10.35(\mathrm{~d}, J=3.4 \mathrm{~Hz}), 10.21$; IR $(\mathrm{KBr}) v\left(\mathrm{~cm}^{-1}\right) 3460.10,3069.21$, 2969.96, 1731.93, 1285.02; HR-MS (ESI): $m / z$ calcd. for $\mathrm{C}_{14} \mathrm{H}_{13} \mathrm{ClFNO}_{4}\left([\mathrm{M}+\mathrm{Na}]^{+}\right) 336.0517$, found 336.0629 .

(E)-6-chloro-3-fluoro-7-hydroxy-4-methyl-2-oxo-2H-chromene-8-carbaldehyde O-butyl oxime (6e): yellow solid; mp $117.2-118.7{ }^{\circ} \mathrm{C}$; yield $21 \%$; ${ }^{1} \mathrm{H}-\mathrm{NMR}\left(400 \mathrm{MHz}, \mathrm{CDCl}_{3}\right) \delta 11.61(\mathrm{~s}, 1 \mathrm{H})$, $8.84(\mathrm{~s}, 1 \mathrm{H}), 7.58(\mathrm{~s}, 1 \mathrm{H}), 4.26(\mathrm{t}, J=6.6 \mathrm{~Hz}, 2 \mathrm{H}), 2.40(\mathrm{~d}, J=2.6 \mathrm{~Hz}, 3 \mathrm{H}), 1.80-1.71(\mathrm{~m}$, $2 \mathrm{H}), 1.46(\mathrm{dd}, J=15.0,7.4 \mathrm{~Hz}, 2 \mathrm{H}), 0.99(\mathrm{t}, J=7.4 \mathrm{~Hz}, 3 \mathrm{H}) ;{ }^{13} \mathrm{C}-\mathrm{NMR}\left(101 \mathrm{MHz}, \mathrm{CDCl}_{3}\right) \delta$ $155.14(\mathrm{~d}, J=2.2 \mathrm{~Hz}), 153.79(\mathrm{~d}, J=30.4 \mathrm{~Hz}), 147.20(\mathrm{~d}, J=2.4 \mathrm{~Hz}), 144.65,141.37,130.72(\mathrm{~d}$, $J=13.8 \mathrm{~Hz}), 126.10(\mathrm{~d}, J=6.0 \mathrm{~Hz}), 119.09,112.15,105.99,75.52,30.76,18.98,13.81,10.34(\mathrm{~d}$, $J=3.6 \mathrm{~Hz})$; IR $(\mathrm{KBr}) \vee\left(\mathrm{cm}^{-1}\right) 3496.26,2955.49,2937.11,1748.95,1288.56$; HR-MS (ESI): $\mathrm{m} / \mathrm{z}$ calcd. for $\mathrm{C}_{15} \mathrm{H}_{15} \mathrm{ClFNO}_{4}\left([\mathrm{M}+\mathrm{Na}]^{+}\right)$350.0674, found 350.0816 .

(E)-6-chloro-3-fluoro-7-hydroxy-4-methyl-2-oxo-2H-chromene-8-carbaldehyde O-allyl oxime (6f): white solid; mp 120.2-120.7 ${ }^{\circ} \mathrm{C}$; yield 76\%; ${ }^{1} \mathrm{H}-\mathrm{NMR}\left(400 \mathrm{MHz}, \mathrm{CDCl}_{3}\right) \delta 11.40(\mathrm{~s}, 1 \mathrm{H}), 8.81$ $(\mathrm{s}, 1 \mathrm{H}), 7.55(\mathrm{~s}, 1 \mathrm{H}), 6.03(\mathrm{~m}, J=16.5,10.4,6.0 \mathrm{~Hz}, 1 \mathrm{H}), 5.43(\mathrm{dd}, J=17.2,1.4 \mathrm{~Hz}, 1 \mathrm{H}), 5.37(\mathrm{dd}$, $J=10.4,1.1 \mathrm{~Hz}, 1 \mathrm{H}), 4.72(\mathrm{~d}, J=6.0 \mathrm{~Hz}, 2 \mathrm{H}), 2.39(\mathrm{~d}, J=2.9 \mathrm{~Hz}, 3 \mathrm{H}) .{ }^{13} \mathrm{C}-\mathrm{NMR}(126 \mathrm{MHz}$, $\delta 155.22(\mathrm{~d}, J=2.8 \mathrm{~Hz}), 153.86(\mathrm{~d}, J=30.6 \mathrm{~Hz}), 147.33(\mathrm{~d}, J=2.6 \mathrm{~Hz}), 145.21(\mathrm{~s}), 142.68(\mathrm{~d}$, $J=249.9 \mathrm{~Hz}), 132.71(\mathrm{~s}), 130.83(\mathrm{~d}, J=13.8 \mathrm{~Hz}), 126.37(\mathrm{~d}, J=6.2 \mathrm{~Hz}), 119.93(\mathrm{~s}), 119.22(\mathrm{~s})$, $112.22(\mathrm{~d}, J=2.8 \mathrm{~Hz}), 105.93(\mathrm{~d}, J=1.5 \mathrm{~Hz}), 76.36(\mathrm{~s}), 10.43(\mathrm{~d}, J=3.7 \mathrm{~Hz})$. FTIR $(\mathrm{KBr}) v$ $\left(\mathrm{cm}^{-1}\right)$ 3470.7, 3415.1, 1753.4, 1377.7, 1267.7; HR-MS (ESI): $\mathrm{m} / z$ calcd. for $\mathrm{C}_{14} \mathrm{H}_{11} \mathrm{ClFNO}_{4}$ $\left([\mathrm{M}+\mathrm{H}]^{+}\right)$312.0433, found 312.0433.

(E)-6-chloro-3-fluoro-7-hydroxy-4-methyl-2-oxo-2H-chromene-8-carbaldehyde O-(3-methylbut-2en-1-yl) oxime (6g): white solid; mp $135.7-136.6{ }^{\circ} \mathrm{C}$; yield $55 \% ;{ }^{1} \mathrm{H}-\mathrm{NMR}\left(600 \mathrm{MHz}, \mathrm{CDCl}_{3}\right)$ $\delta 11.52(\mathrm{~s}, 1 \mathrm{H}), 8.79(\mathrm{~s}, 1 \mathrm{H}), 7.54(\mathrm{~s}, 1 \mathrm{H}), 5.48-5.45(\mathrm{~m}, 1 \mathrm{H}), 4.71(\mathrm{~d}, J=7.3 \mathrm{~Hz}, 2 \mathrm{H}), 2.37$ $(\mathrm{d}, J=2.9 \mathrm{~Hz}, 3 \mathrm{H}), 1.81(\mathrm{~s}, 3 \mathrm{H}), 1.78(\mathrm{~s}, 3 \mathrm{H}) .{ }^{13} \mathrm{C}-\mathrm{NMR}\left(151 \mathrm{MHz}, \mathrm{CDCl}_{3}\right) \delta 155.24(\mathrm{~d}$, $J=2.3 \mathrm{~Hz}), 153.80(\mathrm{~d}, J=30.5 \mathrm{~Hz}), 147.27(\mathrm{~d}, J=2.3 \mathrm{~Hz}), 144.84(\mathrm{~s}), 142.00(\mathrm{~d}, J=56.4 \mathrm{~Hz})$, 140.38 (s), $130.68(\mathrm{~d}, J=13.7 \mathrm{~Hz}), 126.12(\mathrm{~d}, J=6.0 \mathrm{~Hz}), 119.12(\mathrm{~s}), 118.66(\mathrm{~s}), 112.11(\mathrm{~d}$, $J=2.6 \mathrm{~Hz}), 106.11(\mathrm{~s}), 71.86(\mathrm{~s}), 25.86(\mathrm{~s}), 18.20(\mathrm{~s}), 10.33(\mathrm{~d}, J=3.4 \mathrm{~Hz})$. FTIR $(\mathrm{KBr}) \vee\left(\mathrm{cm}^{-1}\right)$ 3494, 3092.8, 2982.8, 1764.6, 1265.2, 1123.6; HR-MS (ESI): $m / z$ calcd. for $\mathrm{C}_{16} \mathrm{H}_{15} \mathrm{ClFNO}_{4}$ $\left([\mathrm{M}+\mathrm{H}]^{+}\right)$340.0746, found 340.0749 .

(E)-6-chloro-3-fluoro-7-hydroxy-4-methyl-2-oxo-2H-chromene-8-carbaldehyde O-prop-2-yn-1-yl oxime (6h): white solid; mp 157.2-158.0 ${ }^{\circ} \mathrm{C}$; yield 67\%; ${ }^{1} \mathrm{H}-\mathrm{NMR}\left(600 \mathrm{MHz}, \mathrm{CDCl}_{3}\right) \delta 11.15$ $(\mathrm{s}, 1 \mathrm{H}), 8.79(\mathrm{~s}, 1 \mathrm{H}), 7.51(\mathrm{~s}, 1 \mathrm{H}), 4.74(\mathrm{~d}, J=2.4 \mathrm{~Hz}, 2 \mathrm{H}), 2.51(\mathrm{t}, J=2.4 \mathrm{~Hz}, 1 \mathrm{H}), 2.31(\mathrm{~d}$, $J=2.9 \mathrm{~Hz}, 3 \mathrm{H}) .{ }^{13} \mathrm{C}-\mathrm{NMR}\left(151 \mathrm{MHz}, \mathrm{CDCl}_{3}\right) \delta 154.21(\mathrm{~d}, J=2.3 \mathrm{~Hz}), 152.70(\mathrm{~d}, J=30.7 \mathrm{~Hz})$, $146.48(\mathrm{~d}, J=2.3 \mathrm{~Hz}), 145.35(\mathrm{~s}), 141.65(\mathrm{~d}, J=250.3 \mathrm{~Hz}), 129.67$ (d, $J=13.7 \mathrm{~Hz}), 125.73(\mathrm{~d}$, $J=6.0 \mathrm{~Hz}), 118.30(\mathrm{~s}), 111.22(\mathrm{~d}, J=2.5 \mathrm{~Hz}), 104.61(\mathrm{~s}), 76.76(\mathrm{~s}), 75.22(\mathrm{~s}), 61.69$ (s), $9.34(\mathrm{~d}$, $J=3.4 \mathrm{~Hz})$. FTIR $(\mathrm{KBr}) v\left(\mathrm{~cm}^{-1}\right) 3476.0,2923.2,1734.8,1261.9$; HR-MS (ESI): $\mathrm{m} / z$ calcd for $\mathrm{C}_{14} \mathrm{H}_{9} \mathrm{ClFNO}_{4}\left([\mathrm{M}+\mathrm{H}]^{+}\right) 310.0277$, found 310.0279 .

(E)-6-chloro-3-fluoro-7-hydroxy-4-methyl-2-oxo-2H-chromene-8-carbaldehyde O-benzyl oxime (6i): white solid; mp 176.8-177.5 ${ }^{\circ} \mathrm{C}$; yield 70\%; ${ }^{1} \mathrm{H}-\mathrm{NMR}\left(600 \mathrm{MHz}, \mathrm{CDCl}_{3}\right) \delta 11.29(\mathrm{~s}, 1 \mathrm{H})$, $8.86(\mathrm{~s}, 1 \mathrm{H}), 7.53(\mathrm{~s}, 1 \mathrm{H}), 7.41(\mathrm{~s}, 2 \mathrm{H}), 7.40(\mathrm{~s}, 2 \mathrm{H}), 7.37-7.35(\mathrm{~m}, 1 \mathrm{H}), 5.23(\mathrm{~s}, 2 \mathrm{H}), 2.36(\mathrm{~d}$, $J=2.9 \mathrm{~Hz}, 3 \mathrm{H}) .{ }^{13} \mathrm{C}-\mathrm{NMR}\left(151 \mathrm{MHz}, \mathrm{CDCl}_{3}\right) \delta 155.21(\mathrm{~d}, J=2.3 \mathrm{~Hz}), 153.78(\mathrm{~d}, J=30.6 \mathrm{~Hz})$, 147.35 (d, J = 2.2 Hz), 145.67 (s), 142.64 (d, J = 250.2 Hz), 135.97 (s), 130.68 (d, J = 13.7 Hz), 128.77 (s), 128.65 (s), 128.55 (s), 126.36 (d, J = 5.9 Hz), 119.17 (s), 112.16 (d, J = 2.5 Hz), 105.92 (s), $77.48(\mathrm{~s}), 10.33(\mathrm{~d}, J=3.4 \mathrm{~Hz})$. FTIR $(\mathrm{KBr}) v\left(\mathrm{~cm}^{-1}\right)$ 3446.2, 1751.0, 1123.0, 1120.3; HR-MS (ESI): $m / z$ calcd. for $\mathrm{C}_{18} \mathrm{H}_{13} \mathrm{ClFNO}_{4}\left([\mathrm{M}+\mathrm{H}]^{+}\right)$362.0590, found 362.0590.

(E)-6-chloro-3-fluoro-7-hydroxy-4-methyl-2-oxo-2H-chromene-8-carbaldehyde O-(4-fluorobenzyl) oxime (6j): white solid; mp 197.1-197.7 ${ }^{\circ} \mathrm{C}$; yield $75 \%$; ${ }^{1} \mathrm{H}-\mathrm{NMR}\left(400 \mathrm{MHz}, \mathrm{CDCl}_{3}\right) \delta$ 
$11.26(\mathrm{~s}, 1 \mathrm{H}), 8.87(\mathrm{~s}, 1 \mathrm{H}), 7.57(\mathrm{~s}, 1 \mathrm{H}), 7.43-7.39(\mathrm{~m}, 2 \mathrm{H}), 7.11(\mathrm{t}, J=8.7 \mathrm{~Hz}, 2 \mathrm{H}), 5.21$ $(\mathrm{s}, 2 \mathrm{H}), 2.39(\mathrm{~d}, J=2.9 \mathrm{~Hz}, 3 \mathrm{H}) .{ }^{13} \mathrm{C}-\mathrm{NMR}\left(151 \mathrm{MHz}, \mathrm{CDCl}_{3}\right) \delta 161.58(\mathrm{~d}, J=31.9 \mathrm{~Hz})$, $155.18(\mathrm{~d}, J=2.3 \mathrm{~Hz}), 153.77(\mathrm{~d}, J=30.6 \mathrm{~Hz}), 147.37(\mathrm{~d}, J=2.2 \mathrm{~Hz}), 145.87(\mathrm{~s}), 142.65(\mathrm{~d}$, $J=250.1 \mathrm{~Hz}), 133.35(\mathrm{~d}, J=39.3 \mathrm{~Hz}), 131.82(\mathrm{~d}, J=3.2 \mathrm{~Hz}), 130.48(\mathrm{~d}, J=8.3 \mathrm{~Hz}), 130.32(\mathrm{~d}$, $J=8.1 \mathrm{~Hz}), 126.48(\mathrm{~d}, J=6.0 \mathrm{~Hz}), 119.19(\mathrm{~s}), 112.21(\mathrm{~d}, J=2.5 \mathrm{~Hz}), 105.84(\mathrm{~s}), 75.61(\mathrm{~s}), 10.34$ $(\mathrm{d}, J=3.4 \mathrm{~Hz})$. FTIR $(\mathrm{KBr}) v\left(\mathrm{~cm}^{-1}\right) 3548.1,3414.9,1736.7,1614.7,1266.7$; HR-MS (ESI): $\mathrm{m} / z$ calcd. for $\mathrm{C}_{18} \mathrm{H}_{12} \mathrm{ClF}_{2} \mathrm{NO}_{4}\left([\mathrm{M}+\mathrm{H}]^{+}\right)$380.0496, found 380.0495.

\subsection{Single Crystal X-Ray Diffraction Analysis}

Fluorinated 7-hydroxycoumarin oxime ether derivatives $(0.1 \mathrm{mmol})$ were dissolved in methanol $(10 \mathrm{~mL})$, then the solution was kept undisturbed at room temperature in the test tubes. Single-crystals suitable for X-ray diffraction were formed within three weeks $[35,36]$. X-ray measurement on the selected crystal was performed on a quartz fiber with protection oil. Cell dimensions and intensities were measured at $296 \mathrm{~K}$ on a Bruker SMART APEX2 CCD area detector diffractometer with graphite monochromated Mo $K \alpha$ radiation $(\lambda=0.71073 \AA)$. The molecular structures were solved via full-matrix least-squares procedure on all the $F^{2}$ data, and the SHELXS-97 and SHELXL-97 programs were employed for structure deal and refinement, respectively. The crystallographic data for the compounds $4 \mathbf{e}, \mathbf{4 h}, \mathbf{5 h}$ and $6 \mathrm{c}$ can be obtained free of charge via http://www. ccdc.cam.ac.uk (or from the CCDC, 12 Union Road, Cambridge CB2 1EZ, UK; E-mail: deposit@ccdc.cam.ac.uk).

\subsection{Antifungal Bioassay}

The test strains Botrytis cinerea (B. cinerea), Alternaria solani (A. solani), Gibberella zeae (G. zeae), Rhizoctorzia solani (R. solani), Colletotrichum orbiculare (C. orbiculare) and Alternaria alternata (A. alternata) were provided by the State \& Local Joint Engineering Research Center of Green Pesticide Invention and Application at Nanjing Agricultural University. The antifungal activities of target compounds were implemented at an equivalent concentration of $100 \mu \mathrm{g} / \mathrm{mL}$ using mycelia growth inhibitory rate methods according to our published literature [16,37]. Every title compound $(30.0 \mathrm{mg}$ ) was dissolved in $0.6 \mathrm{~mL}$ dimethyl sulfoxide and evenly mixed with $299.4 \mathrm{~mL}$ of PSA (potato sucrose agar) medium. An equal volume of methanol in $299.4 \mathrm{~mL}$ medium was used as the blank control. Meanwhile, the Osthole and Azoxystrobin were tested as the positive control fungicides at the same concentration. It carried out on a $100 \mathrm{~mm} \times 15 \mathrm{~mm}$ Petri plate under a sterile condition with 3 replicates. The fungi were inoculated to the center of the medium and cultured at $25{ }^{\circ} \mathrm{C}$ for $2-8$ days in a dark environment. When the mycelia colony of the control grew to $6.5 \mathrm{~cm}(2.5 \mathrm{~d}$ for $B$. cinerea, $7.0 \mathrm{~d}$ for A. solani, $3.5 \mathrm{~d}$ for G. zeae, $1.5 \mathrm{~d}$ for R. solani, $5.0 \mathrm{~d}$ for C. orbiculare and $8.0 \mathrm{~d}$ for A. alternate), the diameters of the mycelium colonies were measured, and the inhibitory percentages were then calculated. The antifungal results of all the compounds were listed in Table 2. The title compounds with inhibition rates exceeding $60 \%$ at $100 \mu \mathrm{g} / \mathrm{mL}$ were further tested for their antifungal effects at five double-declining concentrations (50.00, 25.00, 12.50, 6.25 and $3.125 \mu \mathrm{g} / \mathrm{mL}$ ) for calculating the $\mathrm{EC}_{50}$ values. Similarly, Osthole and Azoxystrobin were employed as positive control fungicides, which were exhibited in Tables 3 and 4 .

\section{Conclusions}

We designed and synthesized three series of fluorinated 7-hydroxycoumarin oxime ether derivatives for the first time. All the target compounds were identified by ${ }^{1} \mathrm{H}-$ NMR, ${ }^{13}$ C-NMR, FTIR and HR-MS. Specifically, the compounds $4 \mathrm{e}, 4 \mathrm{~h}, 5 \mathrm{~h}$ and $6 \mathrm{c}$ were obtained by single-crystal $\mathrm{X}$-ray analysis. The antifungal bioassays in vitro showed that some fluorinated 7-hydroxycoumarin derivatives exhibited a potential biological activity. In particular, the compounds $4 \mathbf{a}, \mathbf{4 f}, \mathbf{5 a}, \mathbf{5 f}, \mathbf{6 a}$ and $\mathbf{6 h}$ were evaluated as the most active ones. The $\mathrm{EC}_{50}$ values of compounds $\mathbf{2 a}, \mathbf{2} \mathbf{b}, \mathbf{4 f}, \mathbf{5} \mathbf{a}$ and $\mathbf{5 f}$ together with Osthole and Azoxystrobin were further tested. The compound $\mathbf{5 f}$ was identified as the promising 
fungicide candidate against $B$. cinerea with the $\mathrm{EC}_{50}$ values of $5.75 \mu \mathrm{g} / \mathrm{mL}$, which was obviously better than Osthole $(33.20 \mu \mathrm{g} / \mathrm{mL})$ and Azoxystrobin $(64.95 \mu \mathrm{g} / \mathrm{mL})$. Additionally, the compound $\mathbf{5 f}$ possessed outstanding antifungal activities against $R$. solani with the $\mathrm{EC}_{50}$ values of $28.96 \mu \mathrm{g} / \mathrm{mL}$, which was superior to Osthole $(67.18 \mu \mathrm{g} / \mathrm{mL})$ and equivalent to Azoxystrobin $(21.34 \mu \mathrm{g} / \mathrm{mL})$. The present work provides a practical foundation for further structural optimization of fluorinated coumarin oxime ether derivatives with the aim to improve the antifungal activity.

Supplementary Materials: The Supplementary Materials containing ${ }^{1} \mathrm{H}-\mathrm{NMR},{ }^{13} \mathrm{C}-\mathrm{NMR}$, FTIR and HR-MS spectra of the title compounds and the crystal structure data of compounds $4 \mathrm{e}, 4 \mathrm{~h}, 5 \mathrm{~h}$ and $6 \mathrm{c}$ can be accessed online.

Author Contributions: W.-H.Z. conceived and designed the experiments; Q.-Q.W. and J.J. carried out the synthesis, characterization and antifungal activity assay of all the compounds; P.D. performed the X-ray analysis; Q.-Q.W. and S.-G.Z. prepared the manuscript for publication; All authors discussed the contents of the manuscript. All authors have read and agreed to the published version of the manuscript.

Funding: This research was funded by National Key R\&D Program of China, grant number 2017YFD0200506 and the Fundamental Research Funds for the Central Universities, grant number KYTZ201604.

Data Availability Statement: The data presented in this study are available on request from the corresponding author.

Conflicts of Interest: The author declare no conflict of interest.

Sample Availability: Samples of the compounds are available from the authors.

\section{References}

1. Li, D.; Zhang, S.; Song, Z.; Wang, G.; Li, S. Bioactivity-guided mixed synthesis accelerate the serendipity in lead optimization: Discovery of fungicidal homodrimanyl amides. Eur. J. Med. Chem. 2017, 136, 114-121. [CrossRef] [PubMed]

2. Wang, X.; Fu, X.; Yan, J.; Wang, A.; Song, Y. Design and synthesis of novel 2-(6-thioxo-1,3,5-thiadiazinan-3-yl)-N' phenylacethydrazide derivatives as potential fungicides. Mol. Divers. 2019, 23, 573-583. [CrossRef]

3. Chen, M.; Zhang, L.; Lu, A.; Wang, X.; Yang, C. Novel carboxylated pyrroline-2-one derivatives bearing a phenylhydrazine moiety: Design, synthesis, antifungal evaluation and 3D-QSAR analysis. Bioorg. Med. Chem. Lett. 2020, 30, 127519. [CrossRef] [PubMed]

4. Ray, M.; Ray, A.; Dash, S.; Mishra, A.; Singh, S. Fungal disease detection in plants: Traditional assays, novel diagnostic techniques and biosensors. Biosens. Bioelectron. 2017, 87, 708-723. [CrossRef]

5. Sparks, T.C.; Lorsbach, B.A. Perspectives on the agrochemical industry and agrochemical discovery. Pest Manag. Sci. 2017, 73, 672-677. [CrossRef]

6. Gobeil-Richard, M.; Tremblay, D.M.; Beaulieu, C.; Van der Heyden, H.; Carisse, O. A pyrosequencing-based method to quantify genetic substitutions associated with resistance to succinate dehydrogenase inhibitor fungicides in Botrytis spp. populations. Pest Manag. Sci. 2016, 72, 566-573. [CrossRef] [PubMed]

7. Hidalgo, W.; Cano, M.; Arbelaez, M.; Zarrazola, E.; Gil, J.; Schneider, B.; Otálvaro, F. 4-Phenylphenalenones as a template for new photodynamic compounds against Mycosphaerella fijiensis. Pest Manag. Sci. 2016, 72, 796-800. [CrossRef] [PubMed]

8. Bizzarri, B.M.; Botta, L.; Capecchi, E.; Celestino, I.; Checconi, P.; Palamara, A.T.; Nencioni, L.; Saladino, R. Regioselective IBX-mediated synthesis of coumarin derivatives with antioxidant and anti-influenza activities. J. Nat. Prod. 2017, 80, 3247-3254. [CrossRef]

9. Bistrović, A.; Stipaničev, N.; Opačak-Bernardi, T.; Jukić, M.; Martinez, S.; Glavaš-Obrovac, L.; Raić-Malić, S. Synthesis of 4aryl-1,2,3-triazolyl appended natural coumarin-related compounds with antiproliferative and radical scavenging activities and intracellular ROS production modification. New J. Chem. 2017, 41, 7531-7543. [CrossRef]

10. Kalaiarasi, G.; Rajkumar, S.R.J.; Dharani, S.; Fronczek, F.R.; Muthukumar Nadar, M.S.A.; Prabhakaran, R. Cyclometallated ruthenium(II) complexes with 3-acetyl-2[H]-chromene-2-one derived CNS chelating ligand systems: Synthesis, X-ray characterization and biological evaluation. New J. Chem. 2018, 42, 336-354. [CrossRef]

11. Hassan, M.Z.; Osman, H.; Ali, M.A.; Ahsan, M.J. Therapeutic potential of coumarins as antiviral agents. Eur. J. Med. Chem. 2016, 123, 236-255. [CrossRef]

12. Zhang, M.Z.; Zhang, R.R.; Wang, J.Q.; Yu, X.; Zhang, Y.L.; Wang, Q.Q.; Zhang, W.H. Microwave-assisted synthesis and antifungal activity of novel fused Osthole derivatives. Eur. J. Med. Chem. 2016, 124, 10-16. [CrossRef]

13. Zhang, M.Z.; Zhang, R.R.; Yin, W.Z.; Yu, X.; Zhang, Y.L.; Liu, P.; Gu, Y.C.; Zhang, W.H. Microwave-assisted synthesis and antifungal activity of coumarin[8,7-e][1,3]oxazine derivatives. Mol. Divers. 2016, 20, 611-618. [CrossRef] [PubMed] 
14. Zhang, R.; Xu, Z.; Yin, W.; Liu, P.; Zhang, W. Microwave-assisted synthesis and antifungal activities of polysubstituted furo[3,2c]chromen-4-ones and 7,8,9,10-tetrahydro-6H-benzofuro[3,2-c]chromen-6-ones. Synth. Commun. 2014, 44, 3257-3263. [CrossRef]

15. Yu, X.; Teng, P.; Zhang, Y.L.; Xu, Z.J.; Zhang, M.Z.; Zhang, W.H. Design, synthesis and antifungal activity evaluation of coumarin-3-carboxamide derivatives. Fitoterapia 2018, 127, 387-395. [CrossRef] [PubMed]

16. Zhang, M.Z.; Zhang, Y.; Wang, J.Q.; Zhang, W.H. Design, Synthesis and antifungal activity of coumarin ring-opening derivatives Molecules 2016, 21, 1387. [CrossRef] [PubMed]

17. Zhang, S.G.; Liang, C.G.; Sun, Y.Q.; Teng, P.; Wang, J.Q.; Zhang, W.H. Design, synthesis and antifungal activities of novel pyrroleand pyrazole-substituted coumarin derivatives. Mol. Divers. 2019, 23, 915-925. [CrossRef]

18. Zhang, R.R.; Liu, J.; Zhang, Y.; Hou, M.Q.; Zhang, W.H. Microwave-assisted synthesis and antifungal activity of novel coumarin derivatives: Pyrano[3,2-c]chromene-2,5-diones. Eur. J. Med. Chem. 2016, 116, 76-83. [CrossRef] [PubMed]

19. Chen, L.Z.; Sun, W.W.; Bo, L.; Wang, J.Q.; Xiu, C.; Tang, W.J.; Shi, J.B.; Zhou, H.P.; Liu, X.H. New arylpyrazoline-coumarins: Synthesis and anti-inflammatory activity. Eur. J. Med. Chem. 2017, 138, 170-181. [CrossRef]

20. Lv, K.; Liu, Y.; Li, Y.; Xu, G.; Pan, X.; Li, F.; Chen, K.; Huang, B.; Yang, Y. Synthesis, fungicidal activity, structure-activity relationship and density functional theory studies of novel oxime ether derivatives containing 1-aryl-3-oxypyrazoles. J. Chem. Res. 2015, 39, 594-600. [CrossRef]

21. Bhargavi, M.V.; Shashikala, P.; Sumakanth, M.; Gunda, S.K. Design, synthesis, molecular docking and biological evaluation of novel coumarin-oxime ether derivatives as COX-2 inhibitors. Asian J. Chem. 2017, 29, 2559-2564. [CrossRef]

22. Joanna, K.; Bartlomiej, P.; Barbara, Z.; Miroslaw, A. Synthesis and biological activity of novel $O$-alkyl derivatives of naringenin and their oximes. Molecules 2017, 22, 1485. [CrossRef]

23. Li, J.; Hu, Y.; Zhang, D.; Liu, Q.; Dong, Y.; Liu, H. Transition metal-catalyzed reactions involving oximes. Adv. Synth. Catal. 2017, 359, 710-771. [CrossRef]

24. Xiao, Y.S.; Yan, X.J.; Xu, Y.J.; Huang, J.X.; Yuan, H.Z.; Liang, X.M.; Zhang, J.J.; Wang, D.Q. Design, synthesis and fungicidal activity of 11-alkoxyimino-5,6-dihydro-dibenzo[b,e] azepine-6-one derivatives. Pest Manag. Sci. 2013, 69, 814-826. [CrossRef]

25. Chen, A.P.; Chen, Y.H.; Liu, H.P.; Li, Y.C.; Chen, C.T.; Liang, P.H. Synthesis and application of a fluorescent substrate analogue to study ligand interactions for undecaprenyl pyrophosphate synthase. J. Am. Chem. Soc. 2002, 124, 15217-15224. [CrossRef] [PubMed]

26. Ferreira, M.L.; Pastoriza-Gallego, M.J.; Araújo, J.M.M.; Canongia Lopes, J.N.; Rebelo, L.P.N.; Piñeiro, M.M.; Shimizu, K.; Pereiro, A.B. The Influence of Nanosegregation on the Phase Behavior of Fluorinated Ionic Liquids. J. Phys. Chem. C 2017, 121, 5415-5427. [CrossRef]

27. Bielenica, A.; Stepien, K.; Sawczenko, A.; Lis, T.; Koziol, A.E.; Madeddu, S.; Collu, D.; Iuliano, F.; Kosmider, A.; Struga, M. Synthesis, structural studies and biological evaluation of halogen derivatives of 1,3-disubstituted thiourea. Lett. Drug Des. Discov. 2017, 14, 1. [CrossRef]

28. Zhang, M.; Yan, X.; Ma, W.; He, Y.; Cai, Z.; Lin, Z. Facile synthesis of tubular magnetic fluorinated covalent organic frameworks for efficient enrichment of ultratrace polybrominated diphenyl ethers from environmental samples. Talanta 2021, $221,121651$. [CrossRef]

29. Chen, Y.; Zhang, Q.; Ma, Y.; Han, Q. Surface-oriented fluorinated pyridinium silicone with enhanced antibacterial activity on cotton via supercritical impregnation. Cellulose 2018, 25, 1499-1511. [CrossRef]

30. Kulkarni, A.; Patil, S.A.; Badami, P.S. Synthesis, characterization, DNA cleavage and in vitro antimicrobial studies of La(III), $\mathrm{Th}(\mathrm{IV})$ and VO(IV) complexes with Schiff bases of coumarin derivatives. Eur. J. Med. Chem. 2009, 44, 2904-2912. [CrossRef]

31. Yan, L.; Zhou, Y.; Du, W.; Kong, Z.; Qi, Z. A new turn on coumarin-based fluorescence probe for Ga ${ }^{3+}$ detection in aqueous solution. Spectroc. Acta Pt. A Mol. Biomolec. Spectr. 2016, 155, 116-124. [CrossRef]

32. Shatzmiller, S.; Bercovici, S. Synthesis and reactions of $\alpha$-Bromo-N-alkoxyimidates. Eur. J. Org. Chem. 1992, 1992, 997-1004. [CrossRef]

33. Wu, J.; Zhang, J.Y.; Gao, P.; Xu, S.L.; Guo, L.N. Copper-catalyzed redox-neutral cyanoalkylarylation of activated alkenes with cyclobutanone oxime esters. J. Org. Chem. 2018, 83, 1046-1055. [CrossRef] [PubMed]

34. Yu, X.Y.; Che, J.R.; Wang, P.Z.; Yang, M.N.; Liang, D.; Xia, W.J. A visible-light-driven iminyl radical-mediated C-C single bond cleavage/radical addition cascade of oxime esters. Angew. Chem. Int. Edit. 2018, 57, 738-743. [CrossRef] [PubMed]

35. Herchel, R.; Travnicek, Z.; Zboril, R. Tuning of the Critical Temperature in Iron (II) Spin-Crossover Materials Based on Bridging Polycyanidometallates: Pentacyanidonitrosylferrate(II) and Hexacyanidoplatinate(IV). Inorg. Chem. 2011, 50, 12390-12392. [CrossRef]

36. Luo, Y.-H.; Wang, J.-W.; Li, Y.-J.; Chen, C.; An, P.-J.; Wang, S.-L.; You, C.-Q.; Sun, B.-W. Selective separation of aqueous sulphate anions via crystallization of sulphate-water clusters. CrystEngComm 2017, 19, 3362-3369. [CrossRef]

37. Liu, Y.; Chen, Z.; Ng, T.B.; Zhang, J.; Zhou, M.; Song, F.; Lu, F.; Liu, Y. Bacisubin, an antifungal protein with ribonuclease and hemagglutinating activities from Bacillus subtilis strain B-916. Peptides 2007, 28, 553-559. [CrossRef] 